\title{
THE UV-OPTICAL GALAXY COLOR-MAGNITUDE DIAGRAM. III. CONSTRAINTS ON EVOLUTION FROM THE BLUE TO THE RED SEQUENCE
}

\author{
D. Christopher Martin, ${ }^{1}$ Ted K. Wyder, ${ }^{1}$ David Schiminovich, ${ }^{2}$ Tom A. Barlow, ${ }^{1}$ Karl Forster, ${ }^{1}$ \\ Peter G. Friedman, ${ }^{1}$ Patrick Morrissey, ${ }^{1}$ Susan G. Neff, ${ }^{3}$ Mark Seibert, ${ }^{1}$ Todd Small, ${ }^{1}$ \\ Barry Y. Welsh, ${ }^{4}$ Luciana Bianchi, ${ }^{5}$ José Donas, $^{6}$ Timothy M. Heckman, ${ }^{7}$ \\ Young-Wook Lee, ${ }^{8}$ Barry F. Madore, ${ }^{9}$ Bruno Milliard, ${ }^{6}$ \\ R. Michael Rich, ${ }^{10}$ Alex S. Szalay, ${ }^{7}$ and Sukyoung K. YI ${ }^{8}$ \\ Received 2006 February 27; accepted 2006 June 18
}

\begin{abstract}
We introduce a new quantity, the mass flux density of galaxies evolving from the blue sequence to the red sequence. We propose a simple technique for constraining this mass flux using the volume-corrected number density in the extinction-corrected UV-optical color-magnitude distribution, the stellar age indexes $H \delta_{A}$ and $D_{n}(4000)$, and a simple prescription for spectral evolution using a quenched star formation history. We exploit the excellent separation of red and blue sequences in the NUV $-r$ band Hess function. The final value we measure, $\dot{\rho}_{T}=0.033 M_{\odot} \mathrm{yr}^{-1} \mathrm{Mpc}^{-3}$, is strictly speaking an upper limit due to the possible contributions of bursting, composite, and extincted galaxies. However, it compares favorably with estimates of the average mass flux that we make based on the red luminosity function evolution derived from the DEEP2 and COMBO-17 surveys, $\dot{\rho}_{R}=+0.034 M_{\odot} \mathrm{yr}^{-1} \mathrm{Mpc}^{-3}$. We find that the blue sequence mass has remained roughly constant since $z=1\left(\dot{\rho}_{B} \simeq 0.01 M_{\odot} \mathrm{yr}^{-1} \mathrm{Mpc}^{-3}\right.$, but the average on-going star formation of $\dot{\rho}_{\mathrm{SF}} \simeq 0.037 M_{\odot} \mathrm{yr}^{-1} \mathrm{Mpc}^{-3}$ over $0<z<1$ is balanced by mass flux off the blue sequence. We explore the nature of the galaxies in the transition zone with particular attention to the frequency and impact of active galactic nuclei (AGNs). The AGN fraction peaks in the transition zone. We find circumstantial, albeit weak evidence that the quench rates are higher in higher luminosity AGNs.
\end{abstract}

Subject headings: galaxies: evolution — ultraviolet: galaxies

\section{INTRODUCTION}

There has been growing interest in the nature of the observed color bimodality in the distribution of galaxies (Balogh et al. 2004; Baldry et al. 2004) that is echoed in other galaxy properties (Kauffmann et al. 2003). The color bimodality is revealed in a variety of color-magnitude plots and is particularly dramatic in the UV-optical color-magnitude diagram (Wyder et al. 2007). The red and blue galaxy concentrations have been denoted the red sequence and the blue "cloud," although we elect to call both concentrations sequences. Deep galaxy surveys are now probing the evolution of the red and blue sequences. Recent work using the COMBO-17 (Bell et al. 2004) and DEEP2 (Faber et al. 2007) surveys provides evidence that the red sequence has grown in mass by a factor of 3 since $z \sim 1$. It is natural to ask what processes have led to this growth, and in particular whether the red

\footnotetext{
1 California Institute of Technology, MC 405-47, 1200 East California Boulevard, Pasadena, CA 91125.

2 Department of Astronomy, Columbia University, New York, NY 10027.

3 Laboratory for Astronomy and Solar Physics, NASA Goddard Space Flight Center, Greenbelt, MD 20771.

4 Space Sciences Laboratory, University of California at Berkeley, 601 Campbell Hall, Berkeley, CA 94720.

5 Center for Astrophysical Sciences, The Johns Hopkins University, 3400 North Charles Street, Baltimore, MD 21218.

${ }^{6}$ Laboratoire d'Astrophysique de Marseille, BP 8, Traverse du Siphon, 13376 Marseille Cedex 12, France.

7 Department of Physics and Astronomy, The Johns Hopkins University, Homewood Campus, Baltimore, MD 21218.

${ }^{8}$ Center for Space Astrophysics, Yonsei University, Seoul 120-749, Korea.

9 Observatories of the Carnegie Institution of Washington, 813 Santa Barbara Street, Pasadena, CA 91101.

${ }^{10}$ Department of Physics and Astronomy, University of California, Los Angeles, CA 90095.
}

sequence has grown via gas rich mergers, gas-less (dry) mergers, or simple gas exhaustion, and whether AGN feedback has played a role in accelerating this evolution (Springel et al. 2005; Di Matteo et al. 2005).

We would like very much to identify galaxies that may be in the process of evolving from the blue to the red sequence. In this paper, we exploit the pronounced demarcation between blue and red sequences afforded by the near-UV (NUV)-optical colormagnitude diagram (Wyder et al. 2007). We make the natural assumption that galaxies residing in the region between the blue and red sequences are making a transition from the former to the latter. We develop a metric (albeit model-dependent) for measuring the speed galaxies are evolving through this transition zone using spectroscopic indices. We use this to estimate the total mass flux of galaxies from the blue to the red sequence during the recent several gigayears. Remarkably, the result we obtain is consistent with estimates based on the evolution of both the red and the blue sequences taken separately.

\section{METHODOLOGY}

We seek to determine the total rate that galaxies residing on the blue sequence evolve to the red sequence. Our approach is to make a series of simplifying assumptions, which we examine in $\S 6$.

1. Galaxies evolving from the blue to the red sequence pass relatively smoothly through intermediate colors. Galaxies do not "jump," for example, by hiding due to strong extinction only to be unveiled as a fully formed red sequence galaxy. In other words, we assume that galaxies transition on timescales no shorter than the precision of the tracers we use below to determine those timescales. 

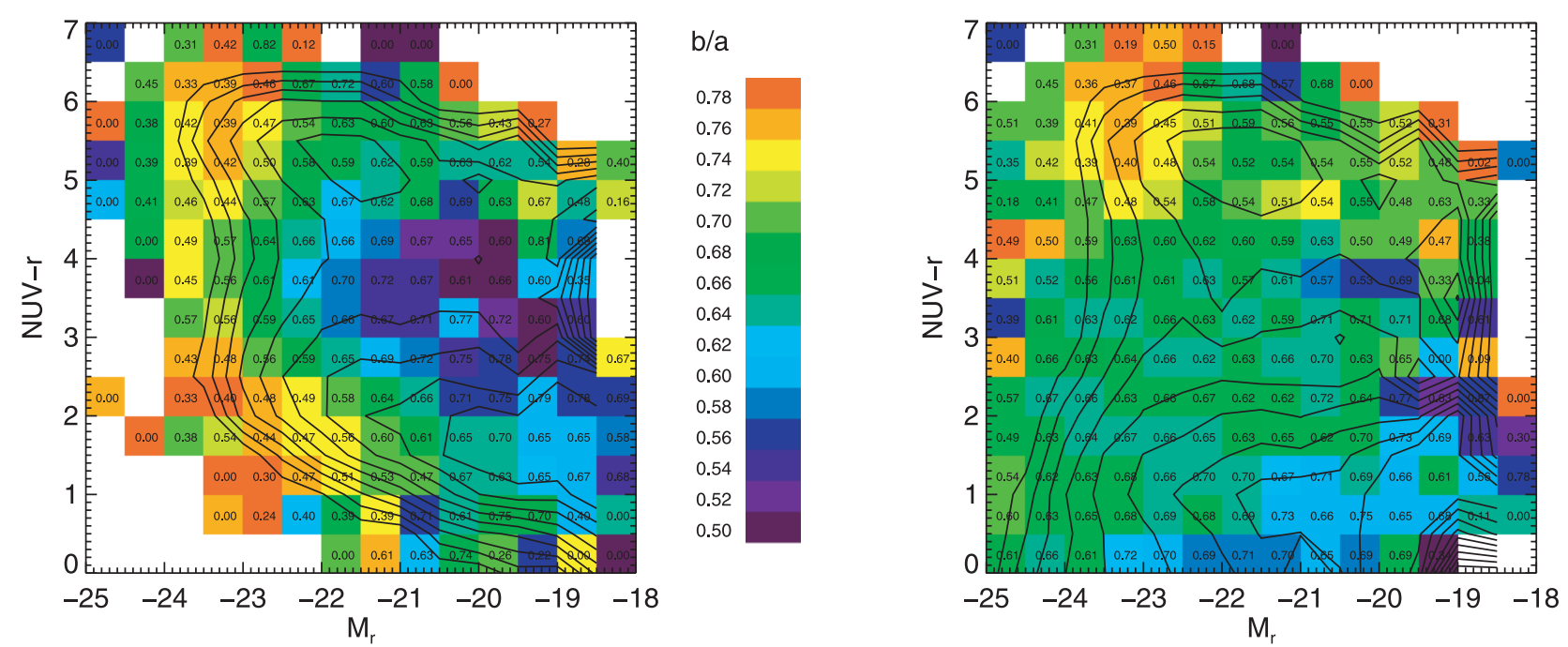

FIG. 1.-Left: Hess diagram with colors indicating mean $b / a$ (minor-to-major axis ratio) for galaxies in each color-magnitude bin. In this and subsequent Hess plots the fractional dispersion in the quantity displayed as a color is given in each bin. The fractional dispersion is the actual dispersion normalized by the range given in the color bar, i.e., 0.4 is $40 \%$ fractional dispersion over a range in $b / a$ of 0.28 , or 0.11 This diagram shows that extinction in edge-on galaxies produces a significant bias in the diagram. Right: Extinction-corrected Hess diagram (using continuum derived $A_{z}$ ) with mean $b / a$ in each bin. Bias observed in the left-hand plot is now virtually eliminated.

2. Galaxies can be described on-average in a given intermediate color-magnitude bin by a single parameterizable star formation history. In other words, we ignore composite stellar populations.

3. We can ignore for the moment the detailed accounting of stellar mass growth due to merging. Rather, our goal is to determine the mass flux off the blue sequence, as a function of stellar mass, and then to total the mass flux leaving the blue sequence.

4. The contribution of galaxies bluing (e.g., due to starbursts) from red to blue sequences through intermediate color bins can be ignored.

With these assumptions, our methodology is quite simple. We define $\dot{\phi}_{B}\left(M_{r}\right)$ as the number flux per unit volume at a given absolute magnitude $M_{r}$ off the blue sequence (unless explicitly stated, the sign of all mass fluxes are defined to be positive). Given that we choose a narrow range of NUV $-r$ color, there is a close cor- respondence between $M_{r}$ and stellar mass. Then the fundamental governing equation is

$$
\phi\left(M_{r}, \mathrm{NUV}-r\right)=\dot{\phi}_{B}\left(M_{r}\right)\left\langle\tau\left(M_{r}, \mathrm{NUV}-r ; \xi\right)\right\rangle,
$$

where $\phi\left(M_{r}, \mathrm{NUV}-r\right)$ is the volume-corrected NUV $-r, M_{r}$ color-magnitude function (Hess function), and $\tau\left(M_{r}, \mathrm{NUV}-r ; \xi\right)$ is the lifetime of the galaxy in this particular color-magnitude bin, for a star formation history $\xi$.

For most physically reasonable star formation histories, the lifetime in the bin is determined by the rate of change of the color, such that

$$
\phi\left(M_{r}, \mathrm{NUV}-r\right) \simeq \frac{\dot{\phi}_{B}\left(M_{r}\right)}{d y / d t(y ; \xi)},
$$
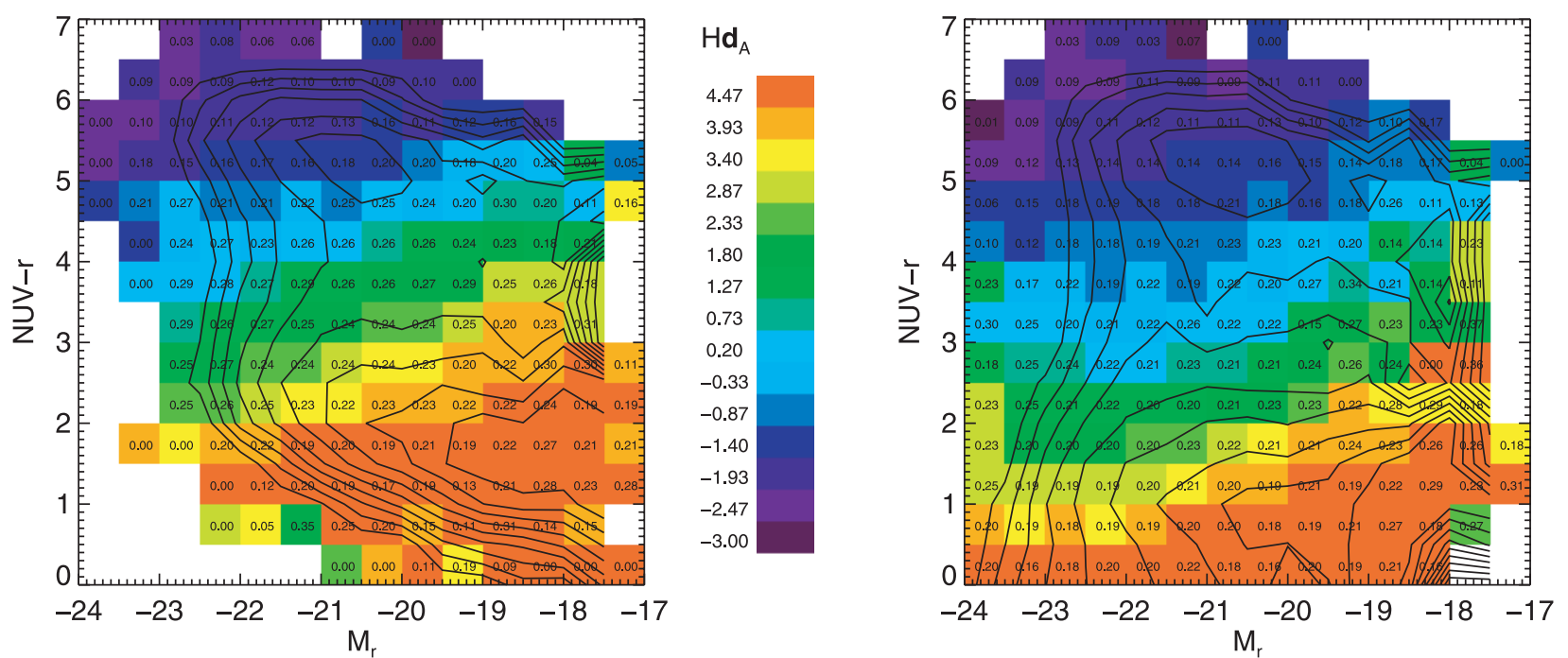

$\mathrm{Hd}_{\mathrm{A}}$

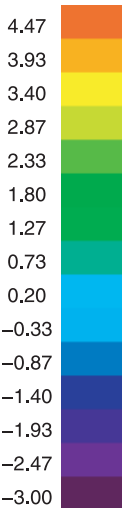

FIg. 2.-Contours show the volume-corrected NUV $-r, M_{r}$ color-magnitude diagram (Hess function). Contours are spaced logarithmically in 10 even steps from $\phi=10^{-5}-10^{-3}\left(0.2\right.$ dex per step). Colors indicate the mean value of $H \delta_{A}$ in each color-magnitude bin. The numeric value in each bin is the fractional standard deviation of $H \delta_{A}$ in each bin, or the standard deviation divided by the typical range of $H \delta_{A}$. Left: no extinction correction; right: extinction correction. 

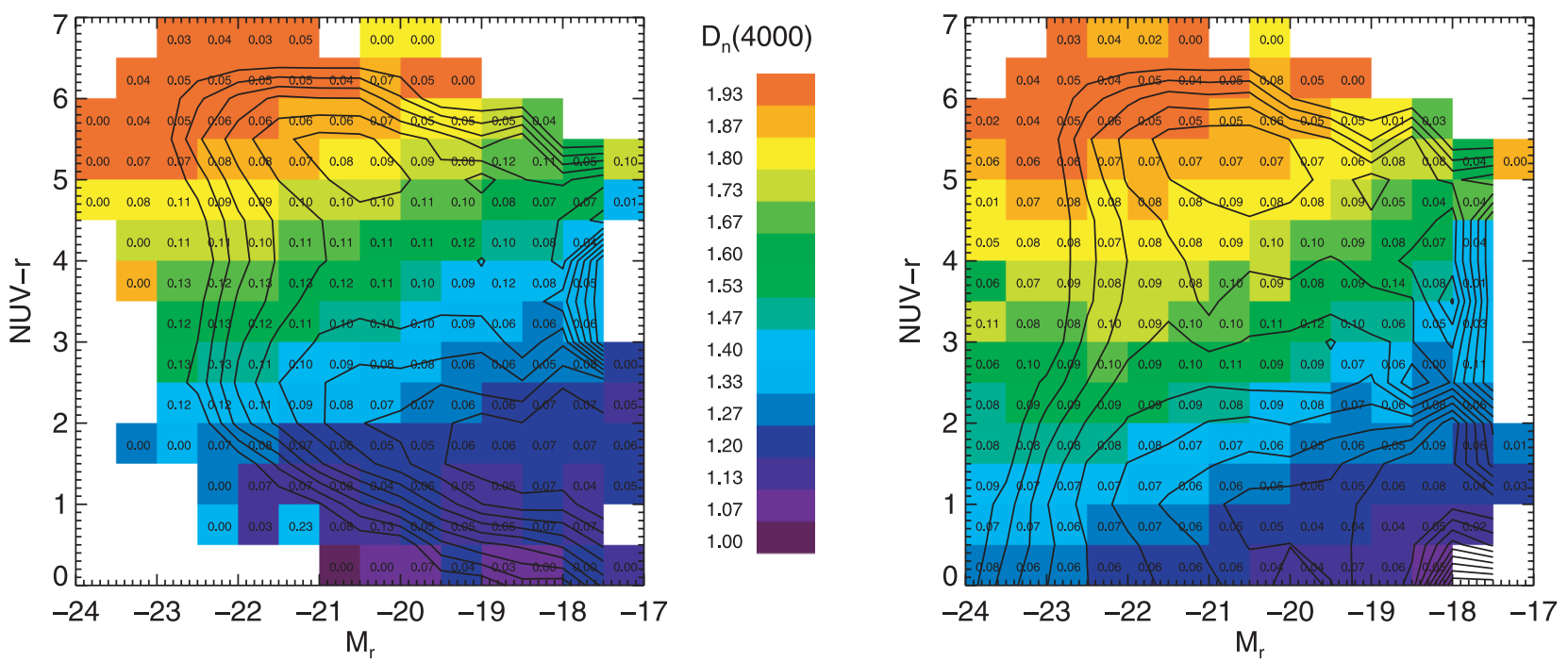

$\mathrm{D}_{\mathrm{n}}(4000)$

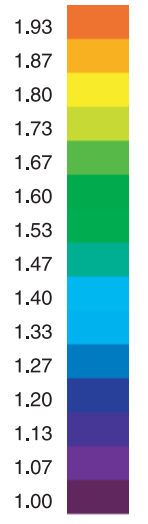

FIG. 3.-Contours show the volume-corrected NUV $-r, M_{r}$ color-magnitude diagram (Hess function). Contours are spaced logarithmically in 10 even steps from

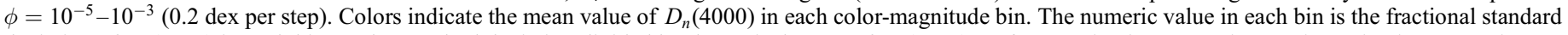
deviation of $D_{n}(4000)$ in each bin, or the standard deviation divided by the typical range of $D_{n}(4000)$. Left: no extinction correction; right: extinction correction.

where $y=\mathrm{NUV}-r ; d y / d t$ is positive due to assumption 4 . The total mass flux is then

$$
\dot{\rho}_{B \rightarrow R} \leq \sum_{M_{r}} M\left(M_{r}\right) \phi\left(M_{r}, \mathrm{NUV}-r\right)\left\langle\frac{d y}{d t}[y ; \xi]\right\rangle,
$$

where $M\left(M_{r}\right)$ is the stellar mass corresponding to $M_{r}$. This equation is an equality only if all galaxies present in the intermediate color bin are moving from the blue to the red sequence. Otherwise it represents an upper limit.

We have determined $\phi\left(M_{r}\right.$, NUV $\left.-r\right)$ in Wyder et al. (2007). The key remaining issue is to determine $d y / d t$. Our approach will be to posit some simple quenched star formation histories and calculate stellar age indexes such as $D_{n}(4000)$ for comparison with the observed galaxy indexes. These age measures will help constrain the family of possible quenching histories at the transitional colors.

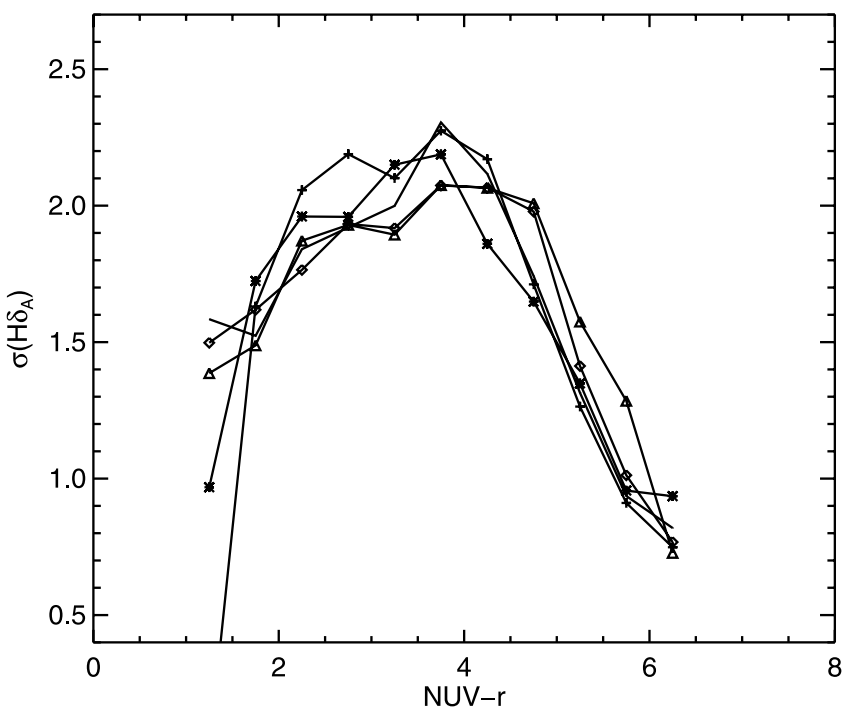

\section{DATA}

\subsection{The Hess Function}

We generate the volume-corrected NUV $-r$ versus $M_{r}$ colormagnitude diagram as discussed in Wyder et al. (2007). We briefly recapitulate the basic method. Our sample is NUV selected in the GALEX Medium Imaging Survey (MIS). The MIS/SDSS DR4 cosample occupies $524 \mathrm{deg}^{2}$ of the north Galactic polar cap and the southern equatorial strip. Our sample is cut as follows: (1) NUV detection, nuv_weight $>800$; (2) SDSS main galaxy sample, $z_{\text {conf }}>0.67$ and specclass $=2$; (3) $14.5<r_{0}<17.6$, $16<$ NUV $<23.0$; (4) nuv_artifact $<2$; (5) field radius less than $0.55^{\circ}$; (6) $0.02<z<0.22$. We generate the volume correction using a simple $V_{\max }$ approach, with joint selection in NUV and $r$ magnitude. The NUV and $r$-band magnitudes are $k$-corrected to $z=0.1$ using the Blanton et al. (2003) code. We have made no evolutionary correction.

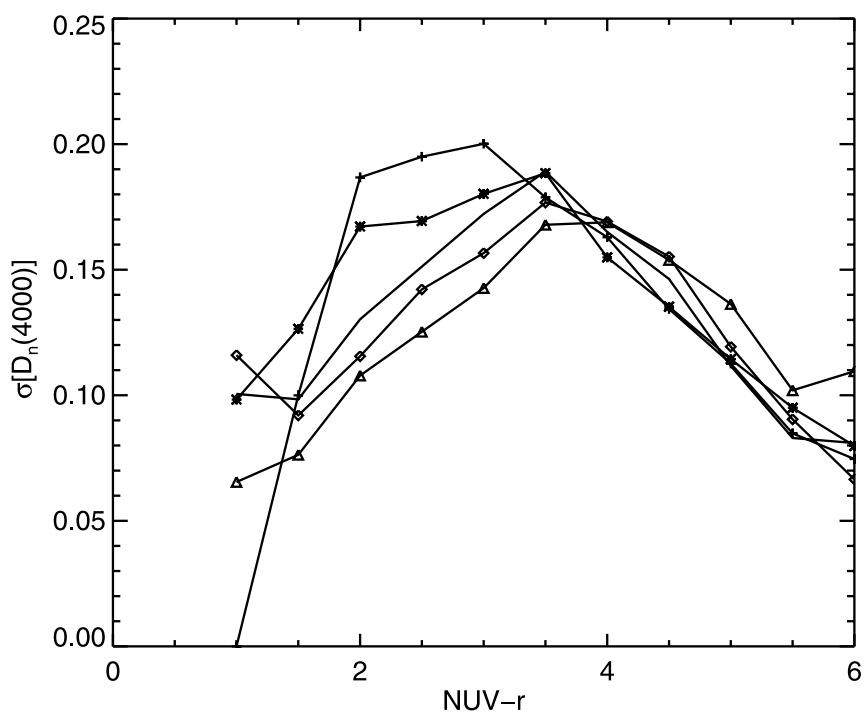

Fig. 4.- Plots show variation of standard deviation from color-magnitude bin means of $H \delta_{A}(l e f t)$ and $D_{n}(4000)(r i g h t)$, vs. NUV $-r$. Symbols give variation with $M_{r}$, with $M_{r}=-22.25,-21.75,-21.25,-20.75$, and -20.25 given by indicated by plus, cross, dot, diamond, and triangle, respectively. 

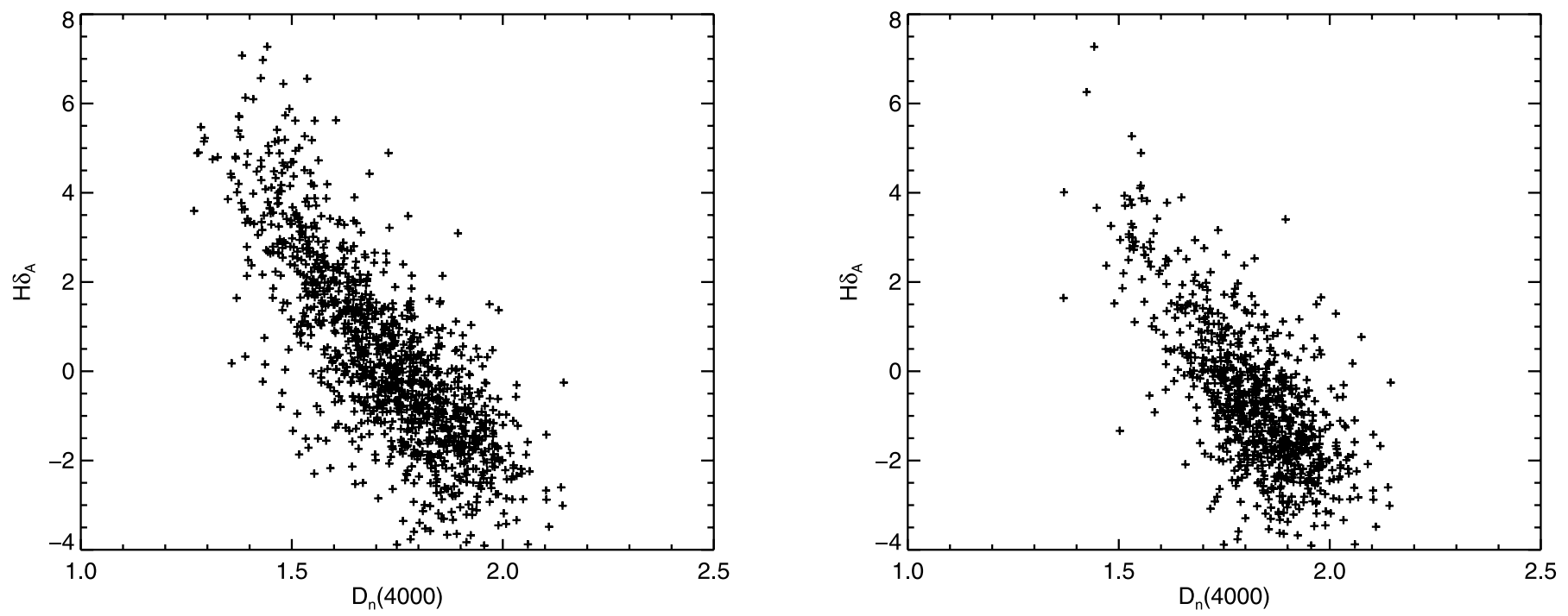

FIG. 5.-Covariance of $H \delta_{A}$ and $D_{n}(4000)$ in a single color-magnitude bin $\left(M_{r}=-21, \mathrm{NUV}-r=4.25\right)$. Left: no extinction correction; right: $A_{z}$ extinction correction.

As shown by Wyder et al. (2007) the Hess function displays a well-defined blue sequence and red sequence. The minimum NUV $-r$ color between the two sequences varies slightly with $M_{r}$, but a good overall choice is NUV $-r \simeq 4$. More precisely, unless otherwise stated, we use the bin with $4.0<\mathrm{NUV}-r<$ 4.5 , or NUV $-r \simeq 4.25$. As we discuss in $\S 6.1 .5$, the results do not depend significantly on this choice.

\subsection{Extinction Corrections}

The NUV $-r$ color of galaxies, and to a lesser extent $M_{r}$, is affected by dust extinction as well as stellar age (e.g., Johnson et al. 2007a, 2007b; Wyder et al. 2007). We therefore construct an extinction-corrected Hess function in order to determine the number density of transition galaxies. We do not use the method of Johnson et al. (2007a, 2007b) because we use the combination of $D_{n}(4000)$ and NUV $-r$ later as a transition rate metric. Kauffmann et al. (2003) measured dust extinction in the $z$ band $\left(A_{z}\right)$ using the continuum fluxes. Brinchmann et al. (2004) measure extinction using the Balmer decrement. Using the derived $A_{z}$ is not ideal because they were derived by comparing $D_{n}(4000)$, $H \delta_{A}$, and the continuum fluxes to a large suite of possible star formation histories. As we discuss in the next section, we also use these indices. We have used both methods to correct the Hess function and find that they yield very similar results (see $\S 6$ ). We discuss there why using the derived $A_{z}$ is a reasonable approach. Our baseline correction uses the $A_{z}$ of Kauffmann et al. (2003). Using a Calzetti et al. (1994) extinction law, we find that $A_{r}=$ $1.6 A_{z}$ and $A_{\mathrm{NUV}}=3.8 A_{z}$.

We show evidence that the extinction correction is working reasonably in Figure 1. This shows the Hess function overlaid with the mean minor-to-major axis ratio for each bin. Without extinction correction (left panel), there is a clear systematic trend of lower inclination galaxies falling in redder NUV $-r$ bins, since lower inclination galaxies will exhibit higher dust extinctions. After the correction (right panel), this trend is almost completely eliminated.

As we see below, the extinction correction has two effects. It reduces the volume density of galaxies in the transition zone. Moreover, it significantly reduces a population of transition color galaxies showing younger than expected spectroscopic ages.

\subsection{Star Formation History Indicators}

We use $D_{n}(4000)$ and $H \delta_{A}$ as calculated and employed for the SDSS spectroscopic sample by Kauffmann et al. (2003) and available as the MPA/JHU DR4 Value-Added Catalog. The value of $H \delta_{A}$ is corrected for nebular emission. We calculate the mean and standard deviation of these quantities (when measured) in each color-magnitude bin of the uncorrected Hess function. In Figures 2 and 3 we show the mean values of $H \delta_{A}$ and $D_{n}(4000)$ in each color-magnitude bin (shown by the bin's color), with the density contours of the Hess function superimposed. We show this for both the observed colors and the extinction-corrected colors. Average values of the two indices transition smoothly from younger to older characteristic ages moving from the blue to the red sequence at a given $M_{r}$. For a given NUV $-r$, ages decrease slowly with decreasing $r$-band luminosity. The relative dispersion of the index within each bin is also shown. Typically, the dispersions are $\sim 0.3$ for $D_{n}(4000$ ) (of the full range of about $1-2.5$ ) and $\sim 2$ for $H \delta_{A}$ (of the full range of -2.5 to 7 in the mean).

The dispersion in both quantities is higher in the transition zone. This is illustrated in Figure 4. In Figure 5 we show that the variance in $H \delta_{A}$ and $D_{n}(4000)$ is correlated as would be expected (and is not due to measurement error). We show these for both the uncorrected and extinction-corrected Hess function. The dispersion is somewhat lower in the extinction-corrected case, as

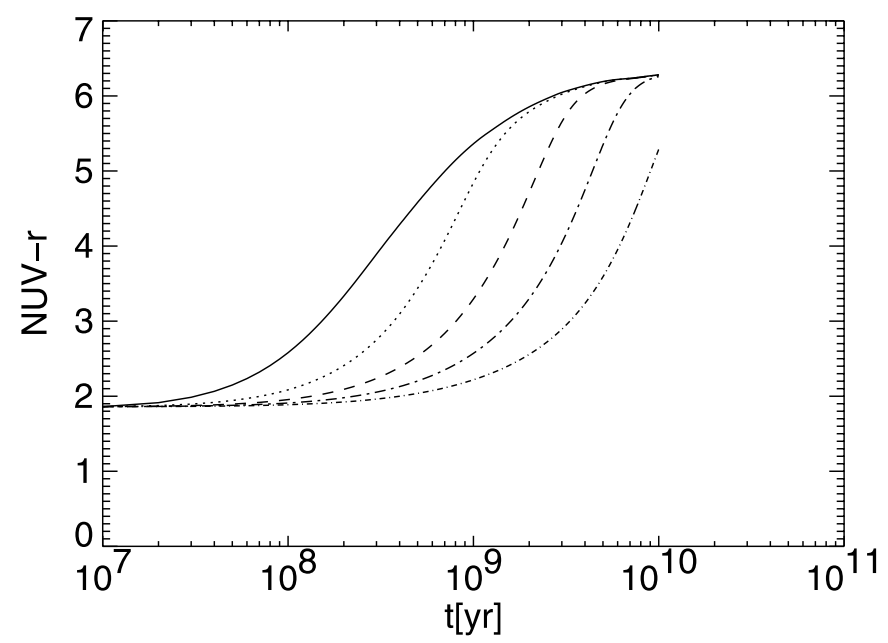

FIG. 6.-Quenched star formation history, NUV $-r$ vs. time after quench. Line style indicates quenching rates: solid line: $\gamma=20 \mathrm{Gyr}^{-1}$; dotted line: $\gamma=$ $5 \mathrm{Gyr}^{-1}$; dashed line: $\gamma=2 \mathrm{Gyr}^{-1}$; dot-long-dashed line: $\gamma=1 \mathrm{Gyr}^{-1}$, dotshort-dashed line: $\gamma=0.5 \mathrm{Gyr}^{-1}$. 

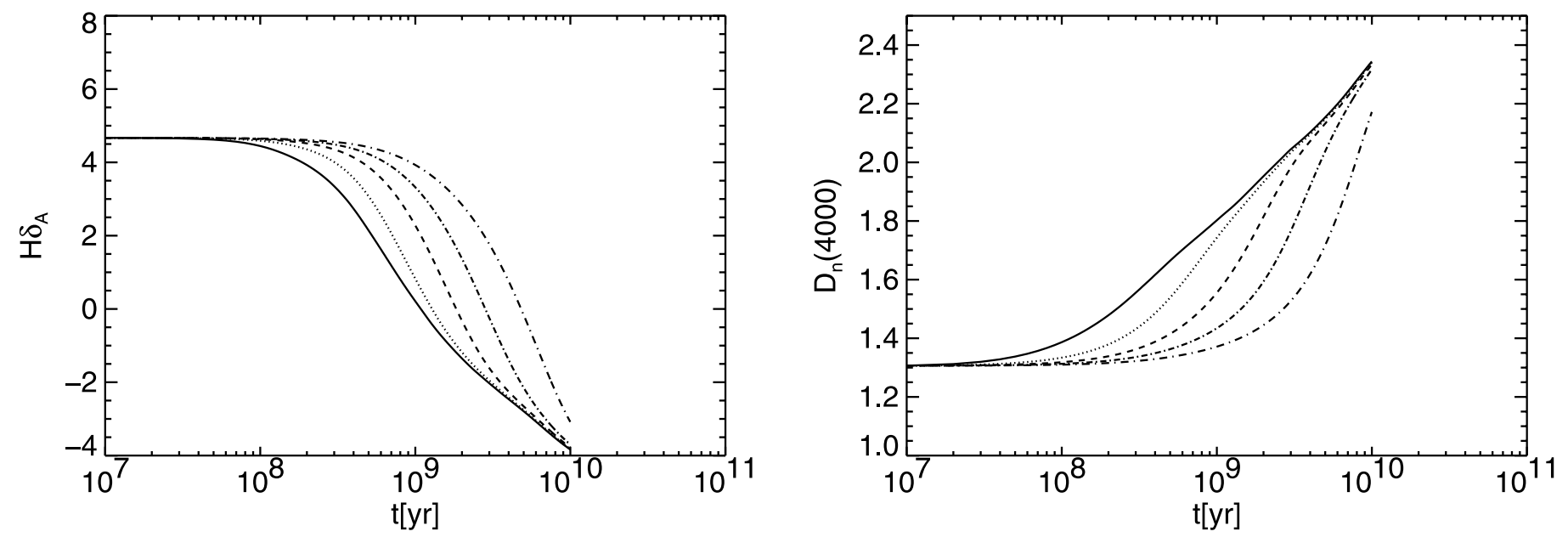

Fig. 7.-Quenched star formation history, $H \delta_{A}($ left $)$ and $D_{n}(4000)$ (right) vs. time after quench. Line style indicates quenching rates: solid line: $\gamma=20$ Gyr ${ }^{-1} ;$ dotted line: $\gamma=5 \mathrm{Gyr}^{-1}$; dashed line: $\gamma=2 \mathrm{Gyr}^{-1}$; dot-long-dashed line: $\gamma=1 \mathrm{Gyr}^{-1}$; dot-short-dashed line: $\gamma=0.5 \mathrm{Gyr}^{-1}$.

some of the lower stellar age galaxies have been shifted to bluer NUV $-r$ bins. The dispersion suggests that we find a range of star formation histories in a given NUV $-r, M_{r}$ color-magnitude bin, even after correction for extinction. We explore this issue in the next section.

\section{QUENCHED STAR FORMATION MODELS}

We seek to explore the rate at which galaxies are transitioning from the blue to the red sequence. In order to complete this analysis, we need to determine the color derivative $d y / d t$ in the transition region. We also hope to gather additional evidence that our star formation history models are a plausible description of the transition galaxies.

Our approach is to use a very simple, parametric model. We assume that star formation on the blue sequence proceeds at a constant rate and is then quenched at a time $t_{q}$. We allow a single free parameter, the quenching rate $\gamma$. Thus, the star formation history we assume is simply

$$
\begin{aligned}
& \dot{M}_{*}=\dot{M}_{*}(0)\left(t<t_{q}\right) \\
& \dot{M}_{*}=\dot{M}_{*}(0)\left(1-e^{-\gamma\left(t-t_{q}\right)}\right)\left(t \geq t_{q}\right) .
\end{aligned}
$$

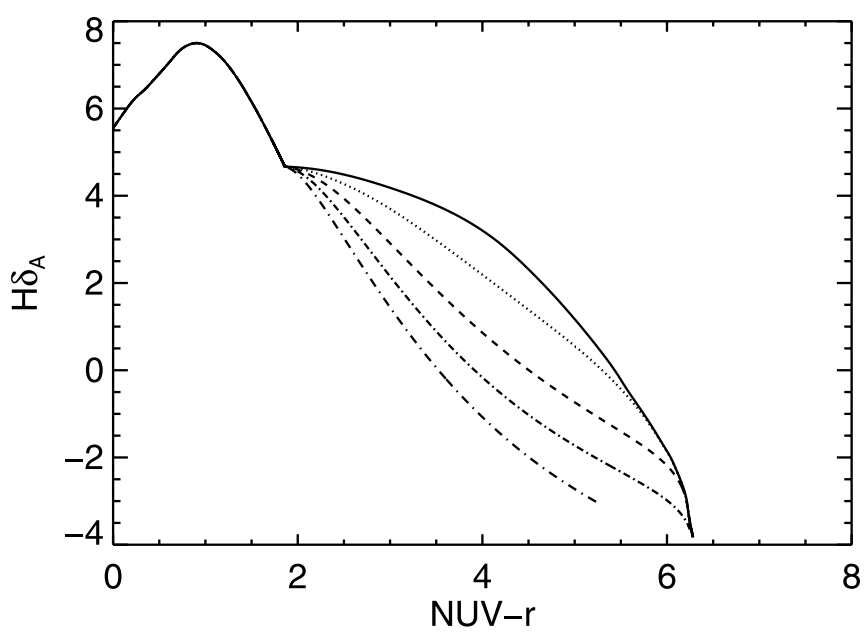

We use the Bruzual \& Charlot (2003) stellar synthesis models to calculate the evolution of the spectral energy distribution. We assume solar metallicity and Salpeter initial mass function. The metallicity assumption is reasonable because, although there is a mass-metallicity variation, the bulk of the mass flux comes from masses $11<\log M<10$, where metallicities are near solar. The small variations around this produce even smaller variations in the model tracks. We also use unextinguished models. We assume that $t_{q}=10 \mathrm{Gyr}$, which is long after all the indexes and colors we consider have reached stable values. We examine below the impact of this assumption.

The evolution of NUV $-r$ for the quenched models is graphed in Figure 6, for a selection of quench rates: $\gamma=$ $[20,5,2,1,0.5] \mathrm{Gyr}^{-1}$, or $\tau=1 / \gamma=[0.05,0.2,1,2]$ Gyr. The transitional color NUV $-r=4.25$ is reached at different times after the quench time $t_{q}$, increasing with $1 / \gamma$.

In order to calculate the indices $H \delta_{A}$ and $D_{n}(4000)$, we use the instantaneous stellar burst index evolution reported by Kauffmann et al. (2003) and compute the continuum weighted average for each star formation history. We find that the indices calculated with the Pickles (1998) stellar library are a better fit to the observed indices at later ages (particularly $H \delta_{A}$ ), so we use these. The evolution of $H \delta_{A}$ and $D_{n}(4000)$ for the quenched models is

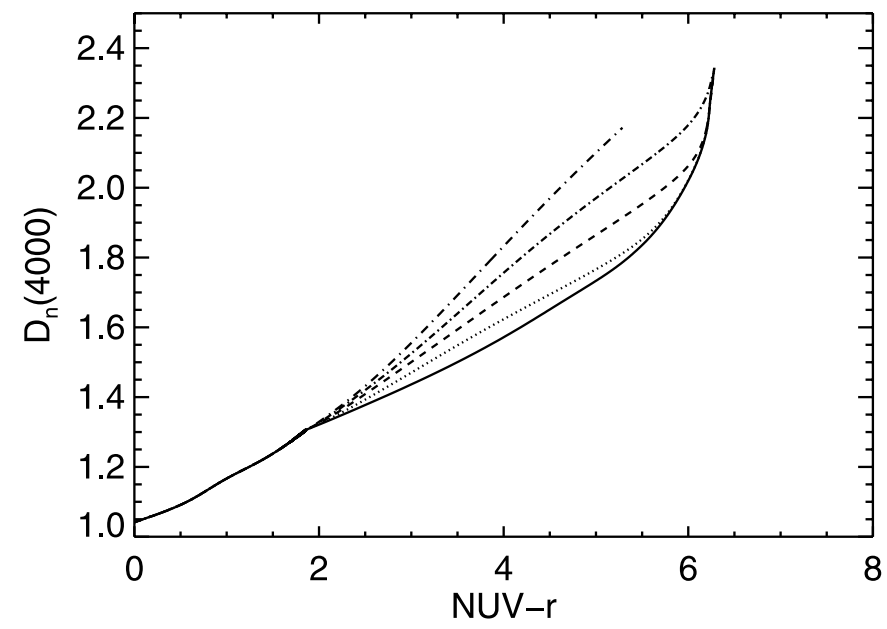

FIG. 8. - Quenched star formation history, $H \delta_{A}($ left $)$ and $D_{n}(4000)($ right $)$ vs. NUV $-r$ after quench. Line style indicates quenching rates: solid line: $\gamma=20$ Gyr ${ }^{-1}$; dotted line: $\gamma=5 \mathrm{Gyr}^{-1}$; dashed line: $\gamma=2 \mathrm{Gyr}^{-1}$; dot-long-dashed line: $\gamma=1 \mathrm{Gyr}^{-1}$; dot-short-dashed line: $\gamma=0.5 \mathrm{Gyr}^{-1}$. 


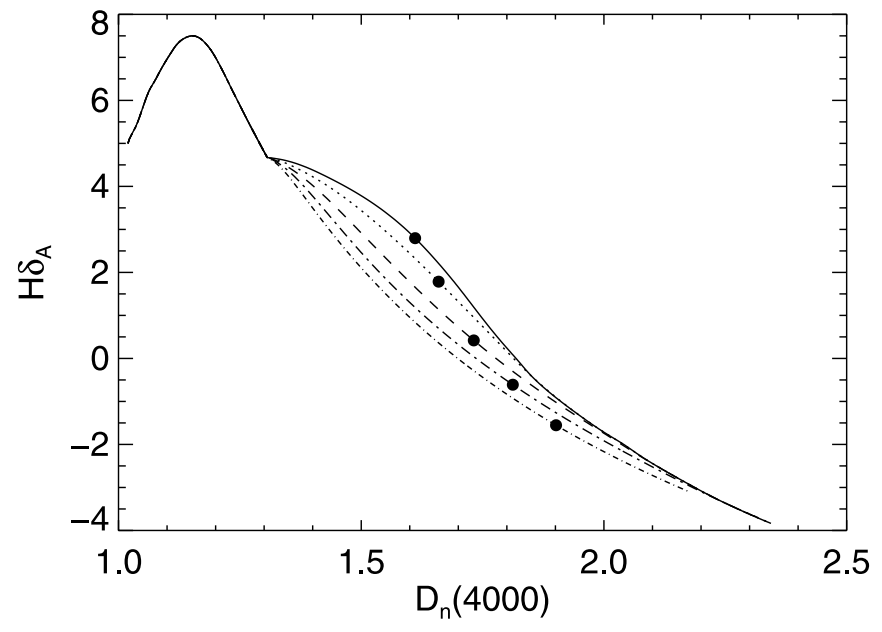

FIG. 9.-Quenched star formation history, $H \delta_{A}$ vs. $D_{n}(4000)$ after quench. Line style indicates quenching rates: solid line: $\gamma=20 \mathrm{Gyr}^{-1}$; dotted line: $\gamma=$ $5 \mathrm{Gyr}^{-1}$; dashed line: $\gamma=2 \mathrm{Gyr}^{-1}$; dot-long-dashed line: $\gamma=1 \mathrm{Gyr}^{-1}$; dot-shortdashed line: $\gamma=0.5 \mathrm{Gyr}^{-1}$. Large points indicate NUV $-r=4.25$ for each $\gamma$.

shown in Figures 7 and 8. This illustrates that for our assumed star formation history, and a fixed NUV $-r$ color, $H \delta_{A}$ and $D_{n}(4000)$ are measures of the quench rate $\gamma$. Faster quench rates yield younger characteristic stellar ages from $H \delta_{A}$ (more positive) and $D_{n}(4000)$ (less positive), at a fixed NUV $-r$ color.

In Figure 9 we show the evolution of $H \delta_{A}$ versus $D_{n}(4000)$ for the various quench rates, and the points on the locus when NUV $-r=4.25$. In Figure 10, we add the galaxies from the extinction-corrected Hess function bin $4<$ NUV $-r<4.5$ and $-22<M_{r}<-21$ (from Fig. 5). Again, we have made no signalto-noise cuts. It can be seen that the model indices are a reasonable representation of the index data and that a range of $\gamma$ can potentially account for much of the dispersion in a given colormagnitude bin.

Finally, in Figure 11, we show the color derivative $d y / d t$ versus color for the quenched models. At $y=4.25$, the derivative varies from a high of $\sim 3 \mathrm{Gyr}^{-1}$ to about $0.4 \mathrm{Gyr}^{-1}$ for $\gamma=0.5 \mathrm{Gyr}^{-1}$. Not surprisingly, $d y / d t(4.25)$ monotonically tracks $\gamma$.

We remind the reader that in the spirit of an exploratory investigation we are using very simple star formation history models to track the evolution in $D_{n}(4000), H \delta_{A}$, and NUV $-r$ space. We are also assuming solar metallicity. The star formation history assumption is further investigated below. We defer a more complex and realistic approach to the future.

\section{CONSTRAINTS ON THE MASS FLUX}

The machinery is now in place to calculate the transitional mass flux. For all calculations we use the color bin $4<y<4.5$. We do this in three ways:

1. Use the uncorrected Hess function, calculate a mean value of $H \delta_{A}$ and $D_{n}(4000)$ for each $M_{r}$ bin, infer the corresponding $d y / d t$ from Figures 10 and 11, and use equation (3) to add up the total mass flux.

2. Use the same methodology as (1), but use the extinctioncorrected Hess function.

3. Calculate a $\gamma$ and $d y / d t$ for each galaxy, and an average $d y / d t$ for each color-magnitude bin.

\subsection{Methods 1 and 2}

In Tables 1 and 2 we tabulate the values of $\phi\left(M_{r}, y=4.25\right)$, $\overline{H \delta_{A}}\left(M_{r}, y=4.25\right),\left\langle D_{n}(4000)\right\rangle\left(M_{r}, y=4.25\right), \gamma, d y / d t$, stellar mass (from Kauffmann et al. [2003] available in the MPIA/JHU Value-Added Catalog), and mass-flux for the uncorrected and extinction-corrected cases. The quench rate $\gamma$ (and the corresponding $d y / d t$ ) are found by finding the $y=4.25\left[D_{n}(4000), H \delta_{A}\right]$ model pair that comes closest to the observed $\left[D_{n}(4000), H \delta_{A}\right]$ pair. Distance in the $\left[D_{n}(4000), H \delta_{A}\right]$ plane is calculated by normalizing each index to its full range, viz., $H \delta_{A n}=H \delta_{A} / 12$, and $D_{n}(4000)($ norm $)=D_{n}(4000) / 1.0$.

In the uncorrected case, the total mass flux is $\dot{\rho}_{T}=0.095 M_{\odot}$ $\mathrm{yr}^{-1} \mathrm{Mpc}^{-3}$ (the sum of the right-hand column). The majority of the mass flux comes from galaxies with $-22<M_{r}<-20$. Lower mass galaxies have younger stellar ages, faster quench rates and larger $d y / d t$. However, their contribution to the total mass is decreased. Note that each mass flux entry in the table is obtained from $\dot{\rho}_{T}=2 M\left(M_{r}\right) \phi\left(M_{r}, y=4.25\right) d y / d t\left[\gamma\left(\left\langle D_{n}(4000)\right\rangle,\left\langle H \delta_{A}\right\rangle\right)\right]$, where the factor of 2 accounts for the fact that the Hess function NUV $-r$ bins are 0.5 wide.

In the extinction-corrected case, the total mass flux is $\dot{\rho}_{T}=$ $0.028 M_{\odot} \mathrm{yr}^{-1} \mathrm{Mpc}^{-3}$, a factor of 2 lower. Two effects cause this: the number density $\phi$ is lower (since some star-forming galaxies are moved to a bluer NUV $-r$ bin), and the characteristic ages are somewhat older (for the same reason). The distribution shifts
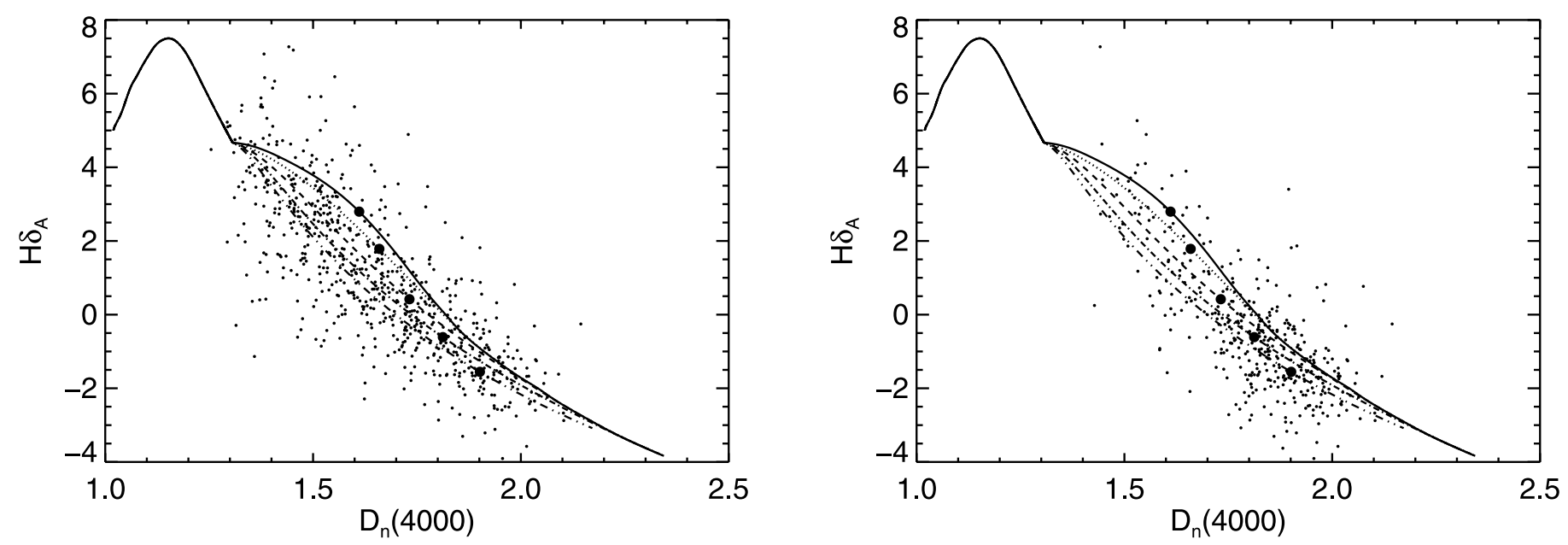

FIG. 10.-Quenched star formation history, $H \delta_{A}$ vs. $D_{n}(4000)$ after quench. Line style indicates quenching rates: solid line: $\gamma=20$ Gyr ${ }^{-1} ;$ dotted line: $\gamma=5$ Gyr ${ }^{-1}$;

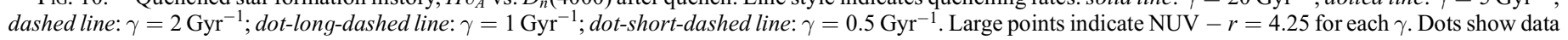
for a single color-magnitude bin $\left(M_{r}=-21\right.$, NUV $\left.-r=4.25\right)$. Left: no extinction correction. Right: extinction corrected. 


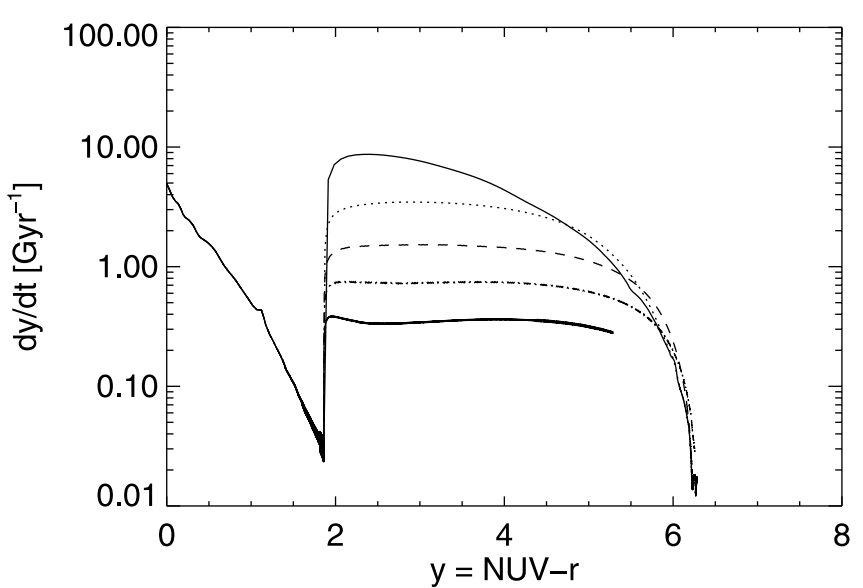

FIG. 11.-Color derivative $d y / d t$ for quenched star formation history vs. NUV $-r$ after quench. Line style indicates quenching rates: solid line: $\gamma=$ $20 \mathrm{Gyr}^{-1}$; dotted line: $\gamma=5 \mathrm{Gyr}^{-1}$; dashed line: $\gamma=2 \mathrm{Gyr}^{-1}$; dot-long-dashed line: $\gamma=1 \mathrm{Gyr}^{-1}$; dot-short-dashed line: $\gamma=0.5 \mathrm{Gyr}^{-1}$.

to higher $M_{r}$ bins, with the major contribution coming from $-23<M_{r}<-20.5$. This second method is likely to be applied in deep galaxy samples - for example, using stacked spectra to study the mass flux evolution.

It is interesting to examine the distribution of galaxies versus $\gamma$. We have binned the galaxies into five $\gamma$ bins, shown in Figure 12. In Figure 13 we show the color derivatives $d y / d t$, the fraction of galaxies in each bin, and the fraction weighted by the color derivative $d y / d t$, which compensates for the briefer residence time of fast quench objects. While the unweighted distribution shows a preponderance of slow-quench galaxies, the weighted distribution shows that the underlying distribution is fairly uniform. Galaxies with faster quench rates will spend less time in the transition region, and the fact that the majority of galaxies are found to have low quench rates in the transition zone does not mean that the majority of galaxies undergo slow quenches.

\subsection{Method 3}

Since there is considerable variance in the age indicators in a given color-magnitude bin, and the nonlinear dependence of $d y / d t$ on the various age indices, we calculate the $\gamma$ and $d y / d t$ for each galaxy and then determine the average $d y / d t$ and its error due to the measurement error of $D_{n}(4000)$ and $H \delta_{A}$, within a particular color-magnitude bin. We perform this average for each $M_{r}$ bin and display the results in Table 3. The color derivatives and resulting mass fluxes are $\sim 15 \%$ larger. The resulting summed mass flux is $\dot{\rho}_{T}=0.033 \pm 0.001 M_{\odot} \mathrm{yr}^{-1} \mathrm{Mpc}^{-3}$. The small reported error is the formal error due to the Poisson error in the Hess function and the index measurement error. The errors in the individual $M_{r}$ bins are given in Table 3 and are typically $\sim 10 \%-25 \%$.

\subsection{Comparison to Mass Growth of the Red Sequence}

Two recent major studies, Bell et al. (2004) using COMBO-17, and Faber et al. (2007) using DEEP2 and COMBO-17, have concluded that the red sequence has grown significantly over the pe$\operatorname{riod} z=1$ to $z=0$. In particular, Faber et al. (2007) measured an increase in the luminosity function normalization of $\Delta \phi_{0}=0.56 \pm$ 0.09 dex since $z=1.0$ and $\Delta \phi_{0}=0.36 \pm 0.09$ dex since $z=0.8$.

We convert this to a mass flux using the following relationship:

$$
\dot{\rho}_{R} \simeq j_{B}(R S)\left(\frac{M}{L_{B}}\right)\left(\frac{10^{\Delta \log \phi_{0}}-1}{10^{\Delta \log \phi_{0}}}\right) \frac{1}{t(z=0.1)-t(z)} .
$$

Faber et al. (2007) estimate that the mass-to-light ratio $M / L_{B}=6$ for today's red sequence galaxies, and the red sequence $B$-luminosity density at $z=0$ is $j_{B}=10^{7.7} L_{\odot} \mathrm{yr}^{-1} \mathrm{Mpc}^{-3}$. This gives a mass flux of $\dot{\rho}_{R}=0.034(0.031) M_{\odot} \mathrm{yr}^{-1} \mathrm{Mpc}^{-3}$ for $0<z<1(0<$ $z<0.8$ ), both with a minimum error of \pm 0.1 dex. By doing this we assume that the mass flux is constant over the redshift interval considered, in order to compare it to the blue sequence mass flux obtained "today" (where for today we use $z=0.1$ ).

We compare these results to the derived blue sequence mass fluxes using method 1 and method 3 in Figure 14. The horizontal bars indicate the redshift range over which the flux has been averaged. For reasons that we discuss in the next section, we consider the blue flux measurement as an upper limit. It is intriguing that the blue sequence and red sequence mass fluxes are in rough agreement. This comparison makes sense only if we are measuring mass fluxes at comparable epochs (in general we are not) or if the mass flux is constant over time (our principal assumption). The overall conclusions of Faber et al. (2007) are not inconsistent with a constant red sequence mass growth rate over $0<z<1$, and although there is some evidence for a slightly increased rate for $0.8<z<1$, the average rate obtained over both redshift intervals is within $10 \%$. We return to this issue in $\S 6$.

\subsection{Comparison to Mass Loss from the Blue Sequence}

To calculate the blue sequence mass flux, we can use the change in the blue luminosity density, but we also need to account for evolution in the mass-to-light ratio. Blanton (2006) has shown by comparing SDSS and DEEP2 luminosity and color-magnitude

TABLE 1

Mass Flux Table: Method 1

\begin{tabular}{|c|c|c|c|c|c|c|c|c|c|}
\hline$M_{r}$ & $\log M_{*}$ & $\phi$ & Number & $\overline{H \delta_{A}}$ & $\overline{D_{n}(4000)}$ & $\gamma$ & $d y / d t$ & $\dot{\phi}$ & $\dot{\rho}_{B}$ \\
\hline$-23.25 \ldots$ & 0.00 & $3.30 \mathrm{E}-08$ & 1 & -1.13 & 1.73 & 1.20 & 0.93 & $1.23 \mathrm{E}-07$ & 0.0000 \\
\hline$-22.75 \ldots \ldots \ldots \ldots \ldots$ & 11.38 & $1.39 \mathrm{E}-06$ & 42 & -0.24 & 1.78 & 1.25 & 0.96 & $5.34 \mathrm{E}-06$ & 0.0006 \\
\hline$-22.25 \ldots \ldots \ldots \ldots \ldots$ & 11.23 & $8.80 \mathrm{E}-06$ & 172 & 0.27 & 1.75 & 1.73 & 1.29 & $4.56 \mathrm{E}-05$ & 0.0039 \\
\hline$-21.75 \ldots \ldots \ldots \ldots \ldots$ & 11.07 & $3.22 \mathrm{E}-05$ & 358 & -0.02 & 1.75 & 1.57 & 1.19 & $1.53 \mathrm{E}-04$ & 0.0089 \\
\hline$-21.25 \ldots \ldots \ldots \ldots \ldots$ & 10.86 & $6.79 \mathrm{E}-05$ & 403 & 0.41 & 1.71 & 2.21 & 1.58 & $4.29 \mathrm{E}-04$ & 0.0156 \\
\hline$-20.75 \ldots \ldots \ldots \ldots \ldots \ldots$ & 10.65 & $9.53 \mathrm{E}-05$ & 304 & 0.63 & 1.70 & 2.58 & 1.77 & $6.76 \mathrm{E}-04$ & 0.0150 \\
\hline$-20.25 \ldots \ldots \ldots \ldots \ldots \ldots$ & 10.47 & $1.25 \mathrm{E}-04$ & 215 & 1.04 & 1.66 & 3.81 & 2.28 & $1.14 \mathrm{E}-03$ & 0.0170 \\
\hline$-19.75 \ldots \ldots \ldots \ldots \ldots$ & 10.26 & $1.33 \mathrm{E}-04$ & 114 & 1.40 & 1.60 & 6.54 & 2.94 & $1.57 \mathrm{E}-03$ & 0.0143 \\
\hline$-19.25 \ldots \ldots \ldots \ldots \ldots$ & 10.04 & $1.13 \mathrm{E}-04$ & 50 & 1.47 & 1.57 & 8.15 & 3.17 & $1.43 \mathrm{E}-03$ & 0.0078 \\
\hline$-18.75 \ldots \ldots \ldots \ldots \ldots$ & 9.77 & $1.64 \mathrm{E}-04$ & 34 & 2.33 & 1.50 & 18.22 & 3.58 & $2.35 \mathrm{E}-03$ & 0.0069 \\
\hline$-18.25 \ldots \ldots \ldots \ldots \ldots$ & 9.42 & $1.01 \mathrm{E}-04$ & 10 & 1.70 & 1.53 & 11.80 & 3.45 & $1.40 \mathrm{E}-03$ & 0.0018 \\
\hline$-17.75 \ldots \ldots \ldots \ldots \ldots$ & 9.37 & $1.88 \mathrm{E}-04$ & 6 & 2.27 & 1.43 & 20.06 & 3.58 & $2.69 \mathrm{E}-03$ & 0.0031 \\
\hline
\end{tabular}


TABLE 2

Mass Flux Table: Method 2

\begin{tabular}{|c|c|c|c|c|c|c|c|c|c|}
\hline$M_{r}$ & $\log M_{*}$ & $\phi$ & Number & $\overline{H \delta_{A}}$ & $\overline{D_{n}(4000)}$ & $\gamma$ & $d y / d t$ & $\dot{\phi}$ & $\dot{\rho}_{B}$ \\
\hline$-23.75 \ldots \ldots \ldots \ldots \ldots \ldots$ & 11.63 & $1.84 \mathrm{E}-07$ & 5 & -0.61 & 1.81 & 0.95 & 0.73 & $5.38 \mathrm{E}-07$ & 0.0001 \\
\hline$-23.25 \ldots \ldots \ldots \ldots \ldots \ldots$ & 11.49 & $1.08 \mathrm{E}-06$ & 22 & -1.05 & 1.85 & 0.72 & 0.53 & $2.30 \mathrm{E}-06$ & 0.0004 \\
\hline$-22.75 \ldots \ldots \ldots \ldots \ldots \ldots$ & 11.32 & $6.53 \mathrm{E}-06$ & 100 & -0.69 & 1.84 & 0.84 & 0.63 & $1.65 \mathrm{E}-05$ & 0.0017 \\
\hline$-22.25 \ldots \ldots \ldots \ldots \ldots \ldots$ & 11.12 & $2.20 \mathrm{E}-05$ & 223 & -0.72 & 1.83 & 0.87 & 0.66 & $5.83 \mathrm{E}-05$ & 0.0038 \\
\hline$-21.75 \ldots \ldots \ldots \ldots \ldots \ldots$ & 10.93 & $4.02 \mathrm{E}-05$ & 259 & -0.66 & 1.82 & 0.91 & 0.69 & $1.11 \mathrm{E}-04$ & 0.0047 \\
\hline$-21.25 \ldots \ldots \ldots \ldots \ldots \ldots$ & 10.73 & $5.63 \mathrm{E}-05$ & 224 & -0.67 & 1.82 & 0.91 & 0.69 & $1.57 \mathrm{E}-04$ & 0.0042 \\
\hline$-20.75 \ldots \ldots \ldots \ldots \ldots \ldots$ & 10.50 & $7.42 \mathrm{E}-05$ & 172 & -0.52 & 1.81 & 0.97 & 0.75 & $2.22 \mathrm{E}-04$ & 0.0035 \\
\hline$-20.25 \ldots \ldots \ldots \ldots \ldots \ldots$ & 10.25 & $6.80 \mathrm{E}-05$ & 81 & -0.18 & 1.76 & 1.41 & 1.08 & $2.94 \mathrm{E}-04$ & 0.0026 \\
\hline$-19.75 \ldots \ldots \ldots \ldots \ldots . .$. & 10.00 & $6.94 \mathrm{E}-05$ & 41 & 0.25 & 1.71 & 2.13 & 1.53 & $4.25 \mathrm{E}-04$ & 0.0021 \\
\hline$-19.25 \ldots \ldots \ldots \ldots \ldots \ldots$ & 9.85 & $6.06 \mathrm{E}-05$ & 22 & -0.19 & 1.73 & 1.64 & 1.24 & $3.01 \mathrm{E}-04$ & 0.0011 \\
\hline$-18.75 \ldots \ldots \ldots \ldots \ldots \ldots$ & 9.52 & $7.22 \mathrm{E}-05$ & 10 & 1.69 & 1.56 & 9.86 & 3.33 & $9.61 \mathrm{E}-04$ & 0.0016 \\
\hline$-18.25 \ldots \ldots \ldots \ldots \ldots \ldots$ & 9.32 & $1.16 \mathrm{E}-04$ & 7 & 0.93 & 1.60 & 5.08 & 2.64 & $1.23 \mathrm{E}-03$ & 0.0013 \\
\hline$-17.75 \ldots \ldots \ldots \ldots \ldots \ldots$ & 9.01 & $1.18 \mathrm{E}-04$ & 2 & 3.01 & 1.44 & 25.94 & 3.51 & $1.66 \mathrm{E}-03$ & 0.0009 \\
\hline
\end{tabular}

distributions that the blue sequence becomes redder by $(u-$ $g)_{0.1}[z=0.1]-(u-g)_{1.0}[z=1] \sim 0.3$ and luminosity decreases by $\sim 1 \mathrm{mag}$. This is consistent with an increase mass-to-light ratio from $\left(M / L_{B}\right)[z=1] \simeq 1.3$ to $\left(M / L_{B}\right)[z=0.1]=2.5$ Bell et al. (2003). Then, with

$$
\dot{\rho}_{B} \simeq \frac{j_{B}(\mathrm{BS})\left(M / L_{B}\right)[z=1]-j_{B}(\mathrm{BS})\left(M / L_{B}\right)[z=0.1]}{t(z=0.1)-t(z)},
$$

and $j_{B}(\mathrm{BS})[z=1]=10^{8.2}$ and $j_{B}(\mathrm{BS})[z=0.1]=10^{7.8}$ for blue sequence galaxies gives $-\dot{\rho}_{B} \simeq 0.01 M_{\odot} \mathrm{yr}^{-1} \mathrm{Mpc}^{-3}$. This mass flux is low, as Blanton (2006) concluded - at least a factor of 4 below our measured transition flux. This conclusion is not changed by extinction, since the extinction vector lies along the $\left(M / L_{B}\right)$ versus $u-g$ locus (Bell et al. 2003).

How can galaxies be populating the transition zone without depleting the mass of the blue sequence? As suggested by the anonymous referee, there is significant mass growth on the blue sequence due to ongoing star formation. If we integrate the star formation history derived, for example, by GALEX measurements

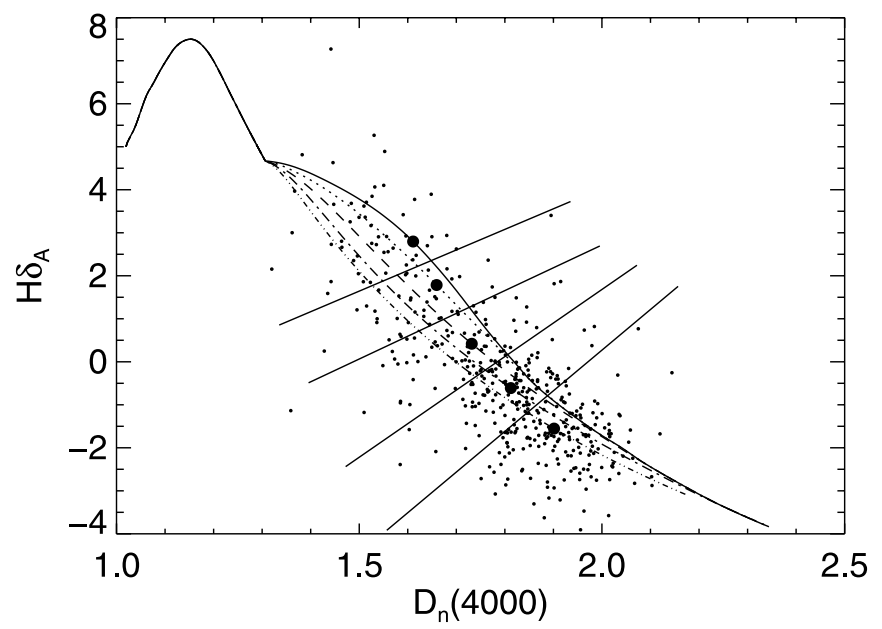

FIG. 12.-Quenched star formation history, $H \delta_{A}$ vs. $D_{n}(4000)$ after quench. Line style indicates quenching rates: solid line: $\gamma=20 \mathrm{Gyr}^{-1}$; dotted line: $\gamma=$ $5 \mathrm{Gyr}^{-1}$; dashed line: $\gamma=2 \mathrm{Gyr}^{-1}$; dot-dashed line: $\gamma=1 \mathrm{Gyr}^{-1}$; triple-dotdashed line: $\gamma=0.5 \mathrm{Gyr}^{-1}$. Large points indicate NUV $-r=4.25$ for each $\gamma$. Dots show data for a single color-magnitude bin $\left(M_{r}=-21\right.$, NUV $\left.-r=4.25\right)$ for the extinction-corrected case. Straight lines indicate bins used to generate Fig. 13.
(Schiminovich et al. 2005) and then (very crudely) divide by the age as in

$$
\dot{\rho}_{\mathrm{SF}} \simeq \frac{\int_{z_{0}}^{z_{1}} \dot{M}_{*}(z) d z}{t\left(z_{0}\right)-t\left(z_{1}\right)},
$$

we find that $\dot{\rho}_{\mathrm{SF}} \simeq 0.037 \pm 0.006 M_{\odot} \mathrm{yr}^{-1} \mathrm{Mpc}^{-3}$ for $z_{0}=0.1$ and $z_{1}=1.0$, and $\dot{\rho}_{\mathrm{SF}} \simeq 0.031 \pm 0.006 M_{\odot} \mathrm{yr}^{-1} \mathrm{Mpc}^{-3}$ for $z_{1}=0.8$.

Remarkably, it appears that on-going star formation balances the mass flux off the blue sequence. In fact, mass continuity, the constancy of the blue sequence mass found by Blanton (2006), and the measured star formation history strongly argue that galaxies are transitioning off the blue sequence at the rates derived independently in this paper. It will be interesting to understand whether this balance is merely a coincidence.

\section{DISCUSSION}

\subsection{Issues and Caveats}

\subsubsection{Aperture Corrections}

SDSS spectroscopy is obtained with $3^{\prime \prime}$ fibers, corresponding to a physical diameter of $3-10 \mathrm{kpc}$ for $0.05<z<0.2$, where the bulk of our sample lies. A possible concern is that this leads to

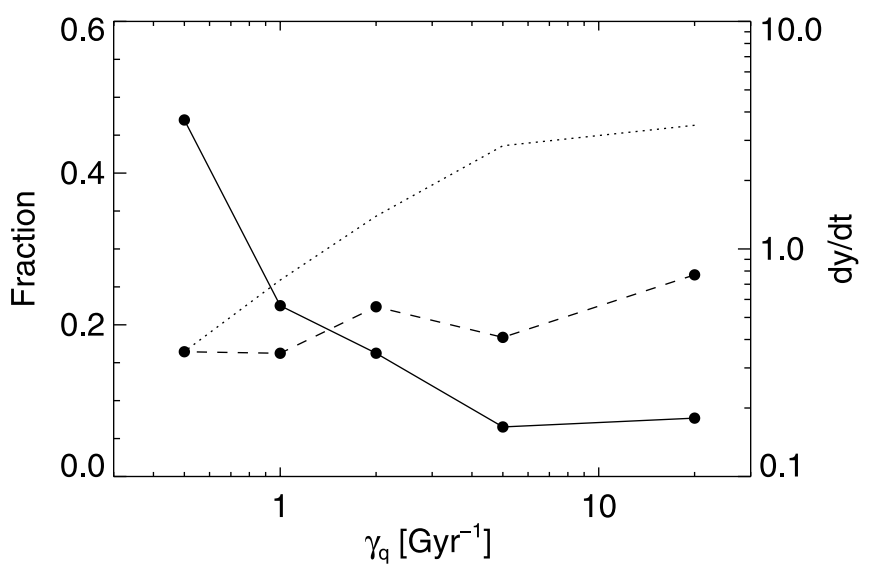

FIG. 13.- Volume-corrected fraction of galaxies in $5 \gamma$ bins shown in Fig. 12. Galaxies have $-23<M_{r}<-20$. Solid line shows unweighted fraction in each bin. Dashed line shows fraction weighted by $d y / d t$, which corrects for the shorter residence time of fast-quenching galaxies. Dotted line shows the color derivative $d y / d t$ in each $\gamma$ bin. 
TABLE 3

Mass Flux Table: Method 3

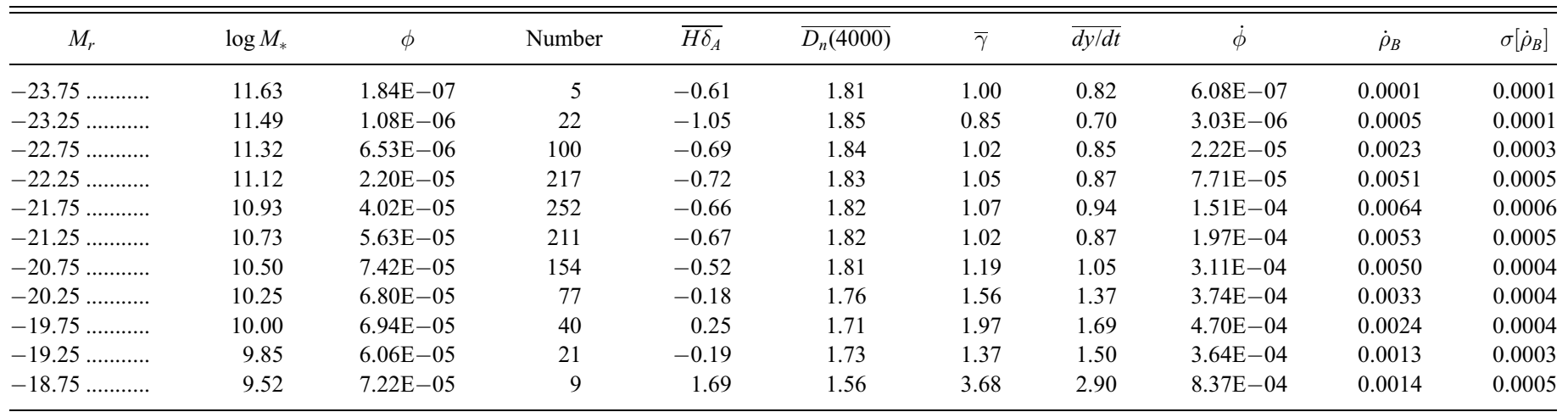

aperture bias. In our case, we might worry that disk light is underrepresented or bulge overrepresented in the $H \delta_{A}$ and $D_{n}(4000)$ indices. This would lead to an underestimate of the quench rate and $d y / d t$. If this were the case, we would expect that the more distant galaxies in the sample would have higher $H \delta_{A}$ [and lower $\left.D_{n}(4000)\right]$ as the disk is brought into the fiber aperture. However, no such trend is apparent in the data - there is no redshift dependence at all in the index values.

\subsubsection{Time Variations and Delays}

In comparing the transitional, red, and blue sequence mass fluxes, we have glossed over time delays in the transition from blue to the red sequence. These could impact the present measurement if there are variations in the mass flux rate over the cosmic timescales spanned by the DEEP 2 and COMBO-17 observations. We have determined typical quench rates to be $\sim 1-1.5 \mathrm{Gyr}^{-1}$. With this rate, evolution from blue to our transitional color takes $\sim 3$ Gyr, with a similar additional interval to reach the red sequence. Strictly speaking, we must compare the mass flux rate now with the blue sequence evolution $3 \mathrm{Gyr}$ in the past and the red sequence evolution $3 \mathrm{Gyr}$ in the future. The results of Faber et al. (2007) suggest that the mass flux rate is roughly constant, although the rate may be higher in the highest redshift bin. It will be of some interest to measure the transitional flux at higher redshifts to determine whether it is evolving with time.

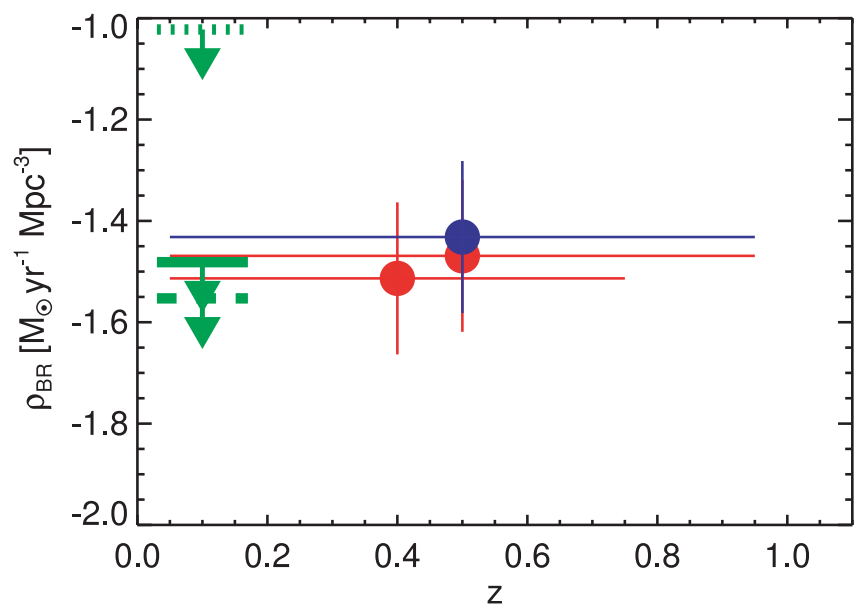

FIG. 14.-Results from this work for the transitional mass flux from the blue to the red sequence. Green dashed line shows the result of method 1, green dotted line method 2, and green solid line method 3. Red points show mass flux estimated from red sequence evolution of Faber et al. (2007). Higher point is based on evolution over $0<z<1$, while lower point on $0<z<0$.8. Blue point shows estimate $-\dot{\rho}_{B}$ based on blue sequence evolution.

\subsubsection{Star Formation Histories}

We have assumed that all galaxies in the transitional region follow the quenched star formation history of equation (4). Many other star formation histories could be considered that would produce different evolution in $H \delta_{A}, D_{n}(4000)$, and color space. We maintain, however, that the quenched models we use lead to the highest possible color derivatives, $d y / d t$, in the transitional region. In this sense, since the mass flux we derive is proportional to $d y / d t$, our result represents an upper limit.

We assumed that the quench occurs after 10 Gyr of constant star formation. This produces some galaxies [with the oldest $D_{n}(4000)$ and $H \delta_{A}$ indices] that exceed the age of the universe by several Gyr. If the constant SFR period is reduced to $5 \mathrm{Gyr}$, the implied quench rate for a given index is lower, and correspondingly the total mass flux is reduced by about $20 \%$ from 0.026 to $0.021 M_{\odot} \mathrm{yr}^{-1} \mathrm{Mpc}^{-3}$ (method 3).

We consider three other possible histories, a smooth exponential decay in star formation rate from formation (smooth), recent bursts, and a composite stellar population, consisting of an early burst and a low level of on-going star formation (composite).

Smooth.-In this case we assume that galaxies were formed $\sim 10$ Gyr ago with a range of $e$-folding parameters $\gamma$ that accounts for the range in present day colors, the original assumption of Tinsley (1968). We show the time evolution of NUV $-r$ in Figure 15. The time at which the transitional color NUV $r=4.25$ is reached depends somewhat on the value of $\gamma$. We show the color evolution of $H \delta_{A}$ and $D_{n}(4000)$ in Figure 16.

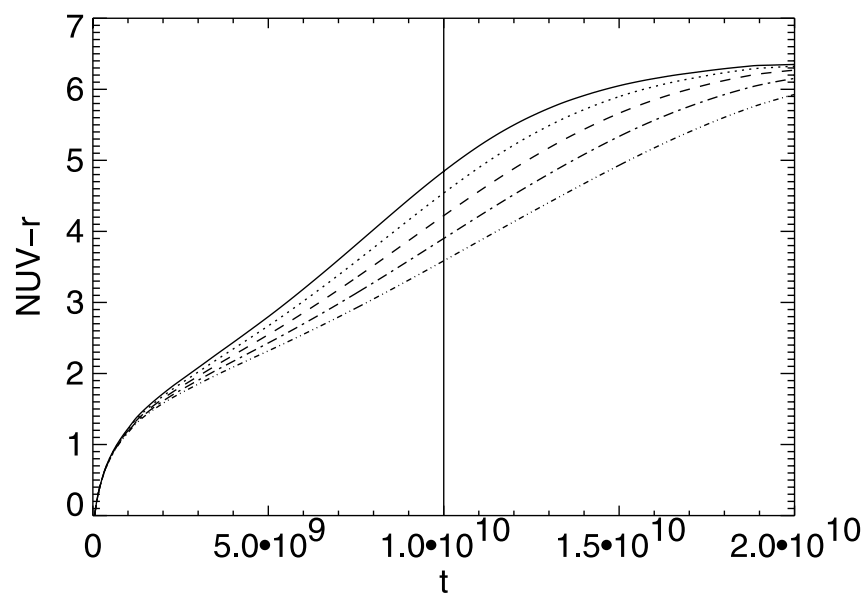

FIG. 15.- Smooth, exponentially decaying star formation rates yield colors NUV $-r=4.25$ near 10 Gyr ages. Line style indicates exponential constant: solid line: $\gamma=0.6 \mathrm{Gyr}^{-1}$; dotted line: $\gamma=0.55 \mathrm{Gyr}^{-1}$; dashed line: $\gamma=0.5 \mathrm{Gyr}^{-1}$; dot-dashed line: $\gamma=0.45 \mathrm{Gyr}^{-1}$; triple-dot-dashed line: $\gamma=0.4 \mathrm{Gyr}^{-1}$. 

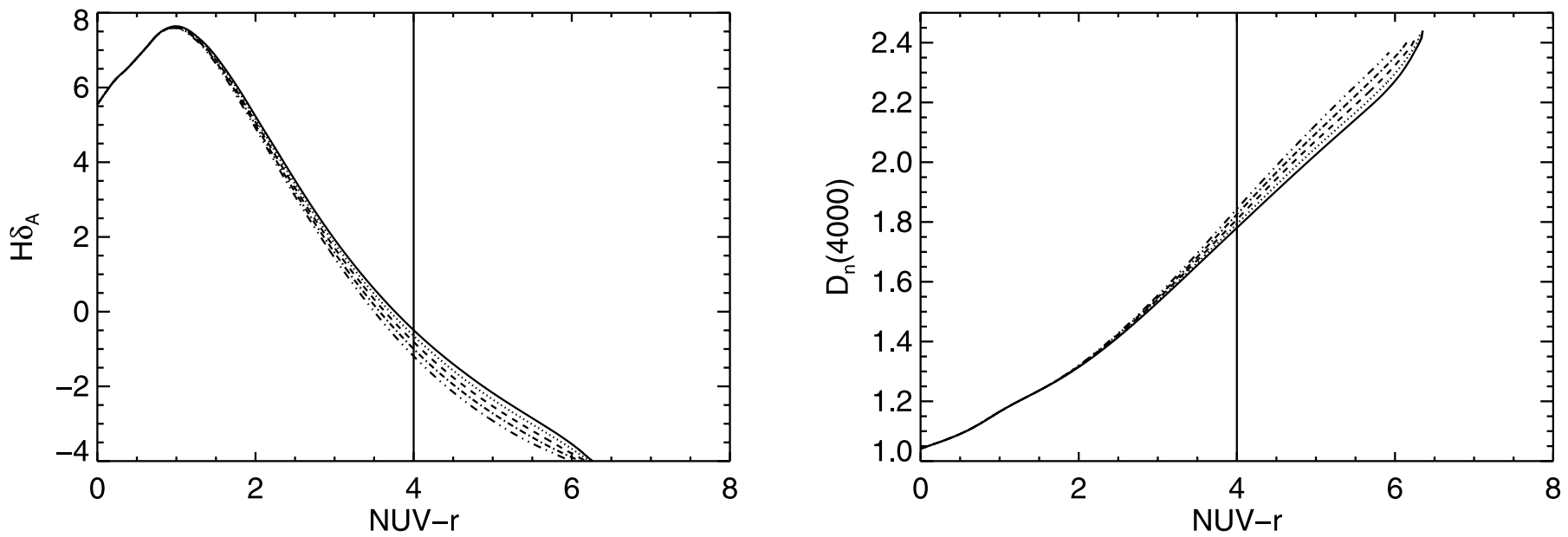

FIG. 16.- Smooth, exponentially decaying star formation rates $H \delta_{A}$ and $D_{n}(4000)$ indices vs. NUV $-r$ color. Line style indicates exponential constant: solid line: $\gamma=0.6 \mathrm{Gyr}^{-1}$; dotted line: $\gamma=0.55 \mathrm{Gyr}^{-1}$; dashed line: $\gamma=0.5 \mathrm{Gyr}^{-1}$; dot-dashed line: $\gamma=0.45 \mathrm{Gyr}^{-1}$; triple-dot-dashed line: $\gamma=0.4 \mathrm{Gyr}^{-1}$.

We show a summary index comparison of the smooth models, quenched models, and data in Figure 17. The smooth models follow roughly the same locus as the slowest quench rate curve $\left(\gamma=0.5 \mathrm{Gyr}^{-1}\right.$ for the quenched model. The points on the locus where NUV $-r$ reaches 4.25 is shown for both model sets. Smooth models reach NUV $-r=4.25$ at "old" index values, corresponding again to the index values at NUV $-r=4.25$ for the $\gamma=0.5 \mathrm{Gyr}^{-1}$ quenched model. The figure also shows the index data (for galaxies in $M_{r}$ bins that contribute the bulk of the mass flux derived above, $-22<M_{r}<-20$ ). The preponderance of galaxies falls at higher $H \delta_{A}$ and lower $D_{n}$ (4000) (above and to the left) than predicted by the smooth models at the transitional color. Many of the galaxies fall above the smooth $H \delta_{A}-$ $D_{n}(4000)$ locus at all colors, where the quenched models provide a better match to both indices at the correct color. For the small fraction of the galaxies have indices and colors consistent with the smooth models, the corresponding color derivative $d y / d t \simeq 0.3$ is very close to that for the low quench rate model with the same

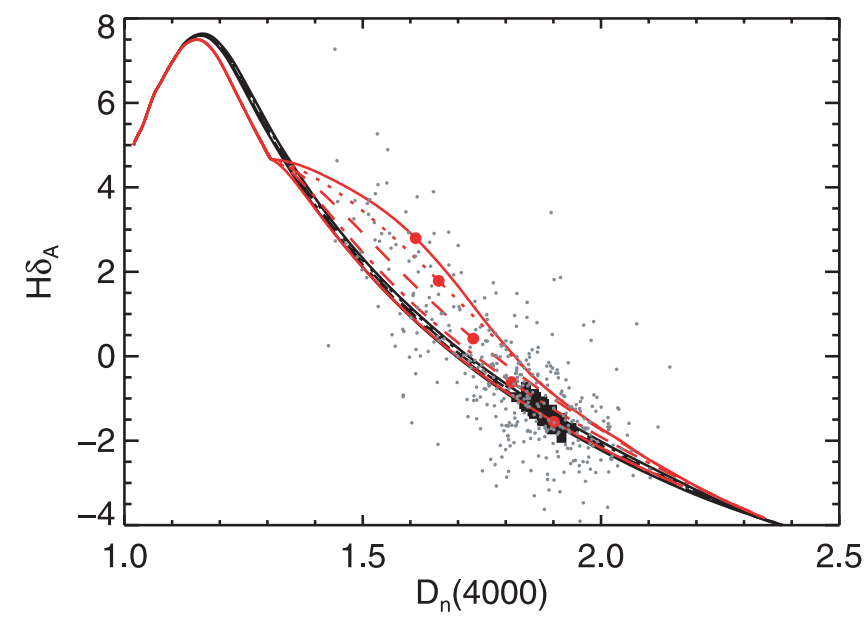

FIG. 17.-Black lines give smooth, exponentially decaying star formation rates $H \delta_{A}$ vs. $D_{n}(4000)$. Line style indicates exponential constant: solid line: $\gamma=0.6 \mathrm{Gyr}^{-1}$; dotted line: $\gamma=0.55 \mathrm{Gyr}^{-1}$, dashed line: $\gamma=0.5 \mathrm{Gyr}^{-1}$; dotdashed line: $\gamma=0.45 \mathrm{Gyr}^{-1}$; triple-dot-dashed line: $\gamma=0.4 \mathrm{Gyr}^{-1}$. Large crosses indicate epoch when NUV $-r=4.25$. In red, quenched models are plotted with the following line styles: solid line: $\gamma=20 \mathrm{Gyr}^{-1}$; dotted line: $\gamma=5 \mathrm{Gyr}^{-1}$; dashed line: $\gamma=2 \mathrm{Gyr}^{-1}$; dot-dashed line: $\gamma=1 \mathrm{Gyr}^{-1}$; triple-dot-dashed line: $\gamma=0.5 \mathrm{Gyr}^{-1}$. Large points indicate epoch when NUV $-r=4.25$ for each $\gamma$. Gray dots are data for $-22<M_{r}<-20$. $\gamma$. In other words, for long decay times, the prior star formation history does not affect the colors and indices: smooth and quenched models give similar results, and our methodology still applies. Clearly, however, the majority of galaxies are not well represented in the index-color diagram with smoothly declining models of age $\sim 10$ Gyr.

Smooth models with significantly higher values of $\gamma$ can produce the transitional colors but only if galaxies continue to form at late times. These models can reproduce the younger spectroscopic ages as well and actually lead to color derivatives and mass fluxes that are not dissimilar to those derived for quenched models. Late formation (rather than late dry merging) of a significant population of red sequence galaxies is not, however, in accord with most observations.

Bursts.-A massive red sequence galaxy suffering a new starburst due to a minor merger with a gas-rich galaxy will evolve from the red to the blue sequence and then may evolve back to the red sequence after the gas supply is exhausted by star formation or feedback. Some fraction of galaxies in the transitional color bins may be in this state moving in either direction. As long as the starburst ramp up time is short, the transition from red to blue will be very fast, with little impact on the transition color

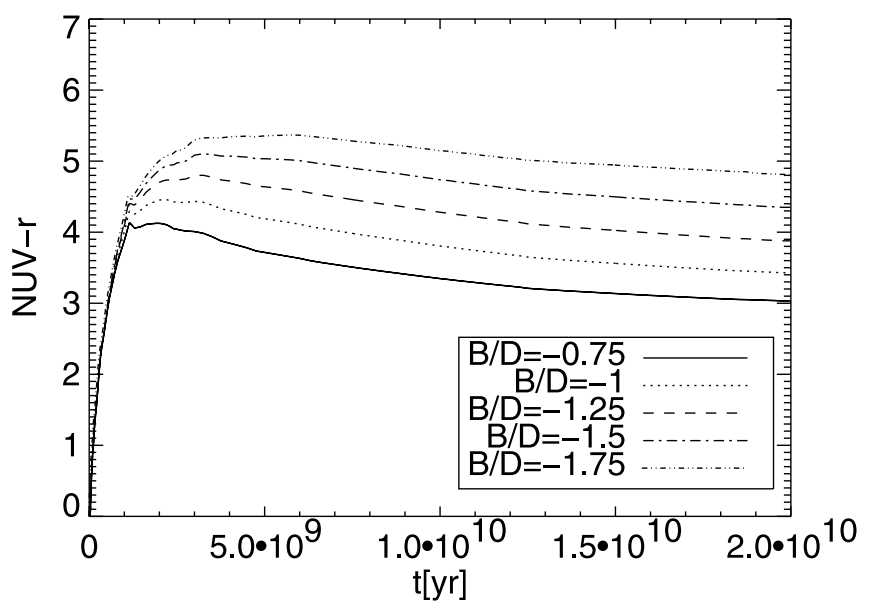

FIG. 18.-NUV $-r$ color vs. time since formation for composite stellar population model with final bulge-to-disk ratio in mass after $20 \mathrm{Gyr}$ given by the legend. The composite model combines a $10^{11} M_{\odot} \tau=0.01$ Gyr exponential bulge with a constant star formation rate disk. The model with $\log B / D=-1.25$ produces galaxies in the transition color NUV $-r=4.25$. 

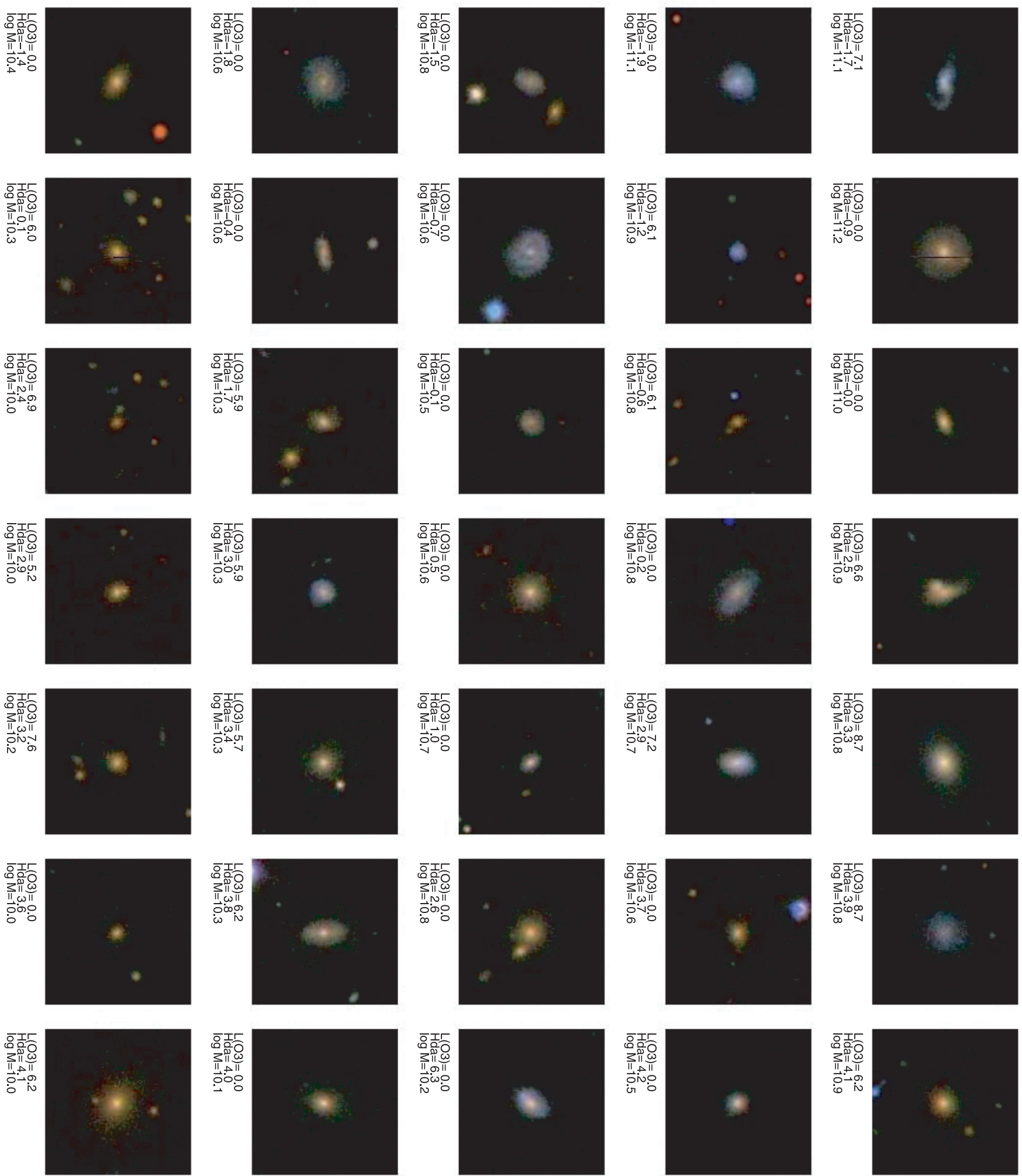

Fig. 19.- - Sample atlas of SDSS $45^{\prime \prime}$ square thumbnails of transition galaxies. Each row is a fixed $M_{r}$ bin, brightest to dimmest going top to bottom, Images are ordered left to right by $H \delta_{A}$ (a proxy in our model for quench rate), with $H \delta_{A}$ (and quench rate) increasing to the right. Log of stellar mass and $H \delta_{A}$ are given.

number densities. However, the subsequent decay is determined again by the "quench time" of the starburst. In general the complexity of possible burst histories makes the present simple treatment inadequate. It can be shown that the quench rate, $H \delta_{A}$ and $D_{n}(4000)$ evolution, and color derivative are similar to that of the quenched case studied above, but with quantitative differences of order $2-3$. There are other subtleties that could influence the derivation of a mass flux from the red sequence evolution of Faber et al. (2007; such as mass-to-light ratio). Regardless, bursting previously red sequence galaxies produce additional numbers 

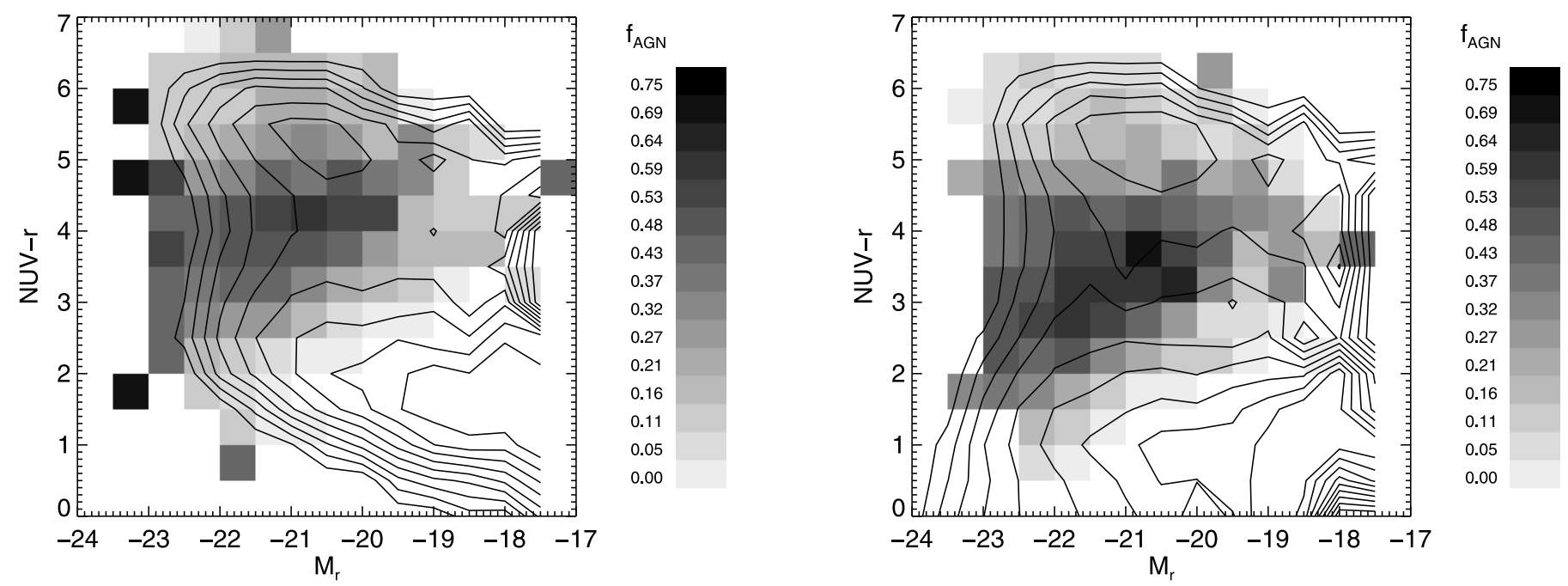

FIG. 20.- Hess function with AGN fraction plotted as gray scale in each bin. Fraction of galaxies in the bin that have AGN or AGN-starburst composite lines based on Heckman et al. (2004). Left: No extinction correction; right: Extinction corrected. Contours are spaced logarithmically in 10 even steps from $\phi=10^{-5}-10^{-3}$ (0.2 dex per step)

in the transitional colors as they return with considerably lower mass flux. We are secure then in interpreting our mass flux measurement as an upper limit.

Composite histories.-An early burst superimposed with lowlevel, on-going star formation can produce a galaxy of any intermediate color if properly tuned. We show this in Figure 18. For example, a $10^{11} M_{\odot}$ bulge can be combined with a $10^{9.75} M_{\odot}$ constant star formation rate disk, which corresponds to ongoing star formation that has produced $3 \%$ of the total mass after $10 \mathrm{Gyr}$, produces a color that hovers around NUV $-r \simeq 4$ after about 10 Gyr. Objects such as this fall on the lower right of the $H \delta_{A}-D_{n}(4000)$ diagram: at the transition color, $H \delta_{A}=-2.1$ and $D_{n}(4000)=1.96$, rather old values. In other words, the relatively low level of ongoing star formation does significantly perturb the spectral indices from that of an old population. The color derivative for this model at the transition color is low and negative, $d y / d t \sim-0.1$. Certainly, there is evidence for lowlevel star formation in nearby ellipticals (Yi et al. 2005). The contribution of galaxies in this bin, as we showed in Figure 13, is relatively small since the color derivative is low anyway. Zeroing this contribution would not significantly affect our result. Alternatively, we can simply note that these galaxies have no impact on our result interpreted as an upper limit.

\subsubsection{Extinction}

We have performed an extinction correction to remove from the transition region heavily reddened blue sequence galaxies. This led to about a factor of 2 decrease in the derived mass flux. The extinctions were obtained for the $z$ band by Kauffmann et al. (2003) using continuum fitting to the star formation histories. The NUV light-weighted stellar age in the quenched models is $\sim 1$ Gyr. It is therefore reasonable to use the $z$-band continuum extinctions in most of these transition color galaxies because the NUV light is tracing stellar populations that have diffused away from star-forming regions. The Kauffmann et al. (2003) suite of star formation histories included smooth declining exponentials plus bursts. Their suite did not explicitly include the kind of quenched models we use, although the combination of bursts and declining models could mimic them. We examined the sensitivity of optical colors to the quench rate and found that, for example, at a fixed (transitional) $g-i$ color there is very little sensitivity to the quench rate in the $D_{n}(4000)$ versus $H \delta_{A}$ plane. In other words, the optical continuum given by smooth exponential models and that produced by our quenched models do not yield significantly different values of $H \delta_{A}$ and $D_{n}(4000)$ since all three are tracing similar timescales. This means that $A_{z}$ derived from the indices and the optical continuum should be reasonably accurate even for quenched models. A self-consistent approach is certainly warranted in a future study. A small fraction of the $A_{z}$ extinctions are negative-for these we assumed zero extinction.

As a check, we repeated the calculation using extinctions obtained by Brinchmann et al. (2004) from the Balmer decrement (and additional lines), again with a Calzetti et al. (1994) extinction law. A significant fraction of the galaxies in the transitional color bins do not have lines strong enough to support this technique. For those we continued to use the continuum-based extinctions. The results of method 3 are virtually identical as are the values of the quenching rates. This is comforting, since the Calzetti et al. (1994) law assumes a particular relationship between $\mathrm{H} \alpha$ and UV extinction that was derived for starburst galaxies.

Removal of more extincted, presumably star-forming, galaxies would likely result in lower values of $\phi\left(M_{r}, \mathrm{NUV}-r\right)$ and $d y / d t$, yielding even lower values of the transitional mass flux.

\subsubsection{Color Variation}

We chose a transitional color bin of $4.0<\mathrm{NUV}-r<4.5$. Is our result dependent on this choice? If our approach is sensible, continuity requires that the mass flux calculated from neighboring color bins should be similar, as long as we are probing truly transitional objects. Differences would arise for two reasons. First, redder transitional colors probe slightly older timescales. If there has been any evolution in the mass flux, then this would produce a trend. It is likely that the mass flux was larger in the past (preliminary analysis using AEGIS data Martin et al. [2008, in preparation] indicates some evolution). Second, objects that reach the red sequence could experience episodic residual star formation events ( Yi et al. 2005) that move them back into the transition region or the bluest part of the red sequence, increasing the apparent mass flux.

We have repeated the analysis, using method 3 , on two neighboring color bins. The results for NUV $-r=3.75,4.25$ [baseline], and 4.75 are, respectively $\dot{\rho}_{T}=0.026,0.033,0.038 M_{\odot}$ 

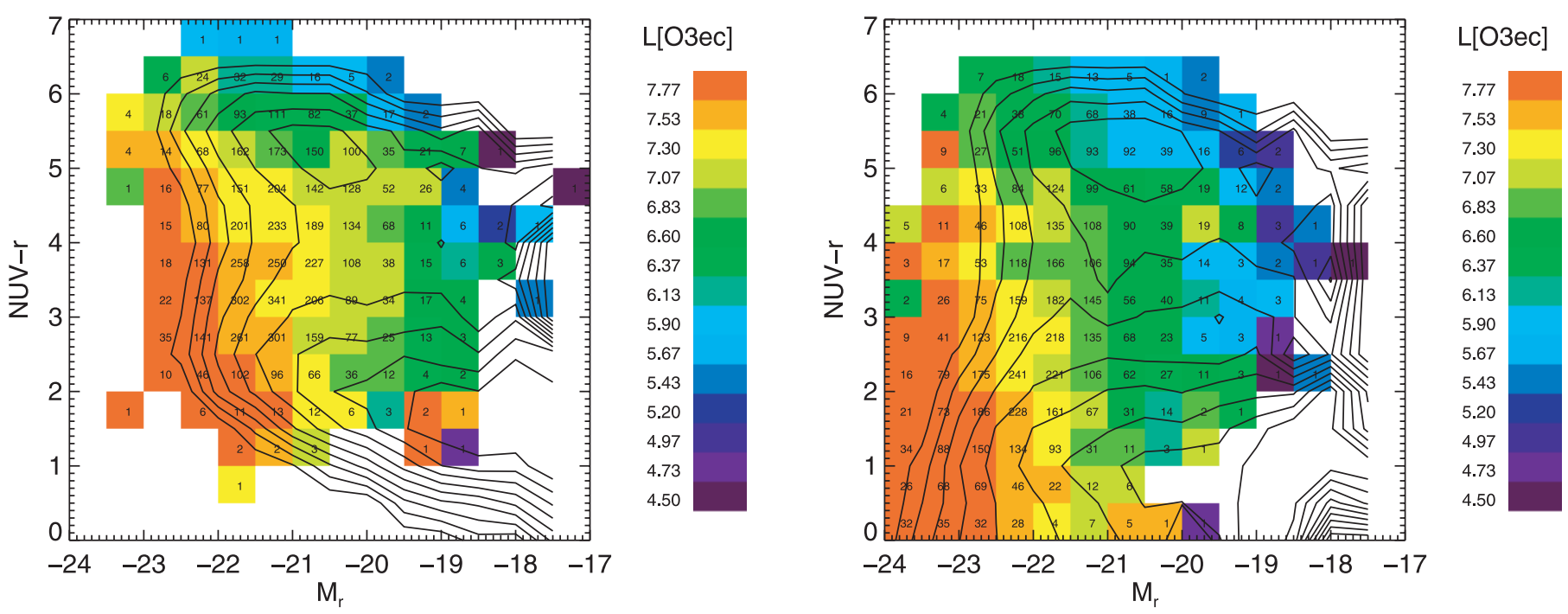

FIG. 21.-Hess function with AGN [O III] luminosity plotted as color map in each bin based on Heckman et al. (2004). Left: No extinction correction; right: Extinction corrected. Contours are spaced logarithmically in 10 even steps from $\phi=10^{-5}-10^{-3}(0.2$ dex per step). Numbers in each bin are number of galaxies.

$\mathrm{yr}^{-1} \mathrm{Mpc}^{-3}$. The trend is consistent with both of the effects discussed above. However, the trend is modest, suggesting that to first order the basic method is sound.

\subsection{Transition Region Galaxies, AGN Feedback, and Mergers}

What kind of galaxies occupy the transitional color region of the Hess function? We show in Figure 19 a small atlas of transitional galaxy thumbnails from the SDSS. The galaxies are organized by mass and $H \delta_{A}$.

One interesting and suggestive property is the preponderance of AGNs. We have used the value-added data set of AGN properties generated by Heckman et al. (2004) to explore this.

We consider two ensemble properties. Heckman et al. (2004) calculated the luminosity in the $\mathrm{O}$ III] line for galaxies that showed line ratios consistent with $\mathrm{AGN}$ or composite $\mathrm{AGN} /$ star formation ionizations. In Figure 20 we show the number fraction of galaxies with some AGN emission in each color-magnitude bin of the Hess function. In Figure 21 we show the mean value of $\left.L\left(\mathrm{O}_{\mathrm{III}}\right]\right)$ for the AGNs in each bin. In each of these cases, although it does not make a significant qualitative difference, we have used the extinction- corrected Hess function. We assume that AGN continuum in these Seyfert II objects is negligible even in the NUV. ${ }^{11}$

Figure 20 is particularly striking, showing that the AGN fraction rises precisely in the transitional color region between the blue and red sequence. This is of course extremely suggestive of an important role for AGN feedback (Springel et al. 2005) in quenching star formation more rapidly than simple gas exhaustion.

In the context of this work, we can ask whether the derived model parameters, in particular the quench rate, is correlated with AGN properties, such as luminosity. In Figure 22, we show for each galaxy, in the range $-23<M_{r}<-20, L(\mathrm{O}$ III $\left.]\right)$ versus $H \delta_{A}$ and the derived quench rate $\gamma$. There is considerable scatter. Linear regression gives for $\left.\log L\left(\mathrm{O}_{\mathrm{III}}\right]\right)=a \log \gamma+b, a=0.37 \pm$ 0.05 , with a correlation coefficient of only 0.32 . The trend is most evident in Figure 23, where the median luminosity is calculated in five $\gamma$ bins.

${ }^{11}$ If this is not the case, some galaxies at transitional colors are red sequence "interlopers." In principal the mass flux could be even lower when these objects are removed, although the correction could also add some galaxies from bluer bins.
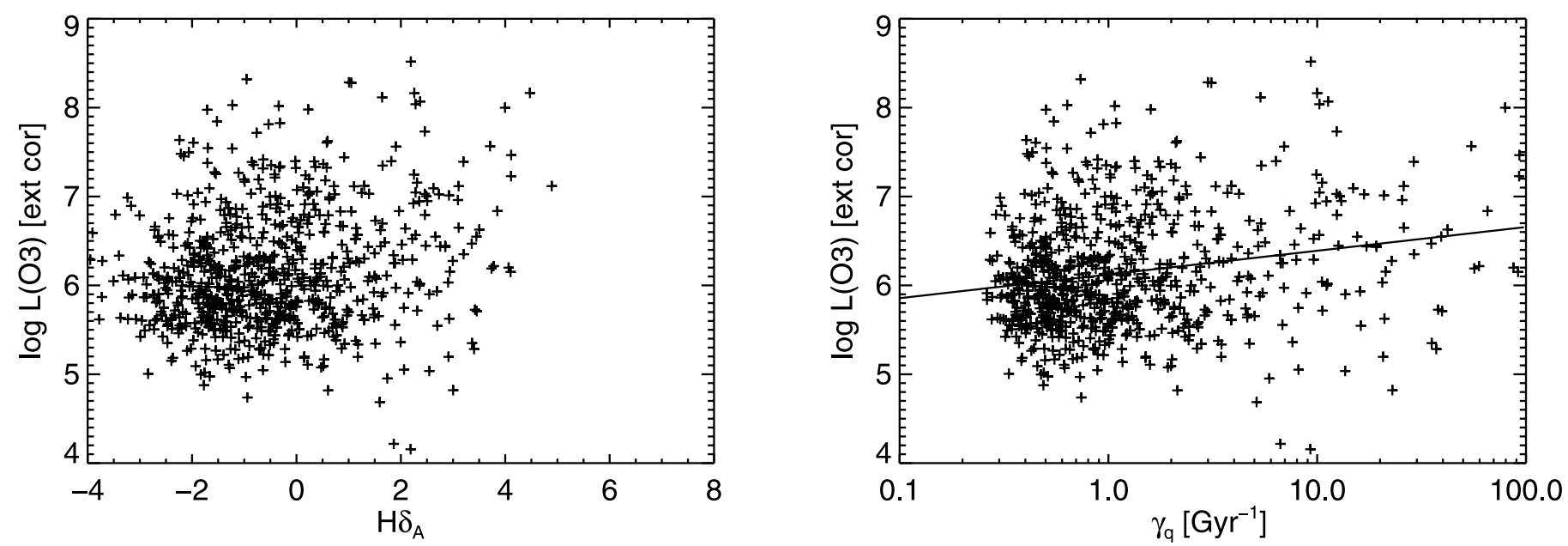

FIG. 22.-We show for each galaxy in the range $-23<M_{r}<-20 L(\mathrm{O}$ III] $)$ vs. $H \delta_{A}(l e f t)$ and the derived quench rate $\gamma(r i g h t)$. Color range is $3.75<\mathrm{NUV}-r<4.75$. 


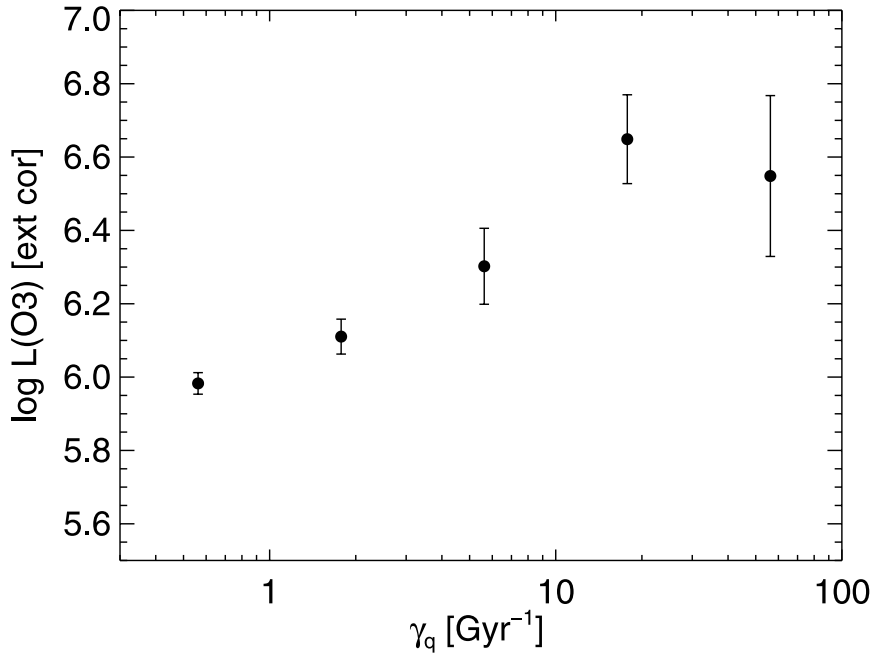

FIG. 23.-Median of $\log L_{[\mathrm{O}}$ III] vs. $\gamma$ in bins of $\Delta \gamma=0.5$. Color range is $3.75<\mathrm{NUV}-r<4.75$. Absolute magnitude range is $-23<M_{r}<-20$.

There is a modest trend of $L(\mathrm{O}$ III $])$ increasing with $\gamma$. Luminosity nominally traces accretion rate, which is likely to be higher in the gassier galaxies nearer the blue sequence. Higher $H \delta_{A}$ galaxies have more recently lost their gas fueling star formation, and there may be a time delay for that to starve the central engine. Thus, the strongest statement that can be made is that galaxies with AGNs of higher luminosity tend to show higher quench rates.

Heckman et al. (2004) computed a specific growth rate for central black holes by using $L(\mathrm{O}$ III] $)$ to obtain a rough bolometric luminosity and the central velocity dispersion and the $M-\sigma$ relation (Tremaine et al. 2002) to obtain the black hole mass. The result is

$$
\left.\frac{\dot{M}_{\mathrm{BH}}}{M_{\mathrm{BH}}}=\gamma_{\mathrm{BH}}=10^{-7.8} L\left(\mathrm{O}_{\mathrm{III}}\right]\right) \sigma_{200}^{4} \mathrm{Gyr}^{-1},
$$

which we compared with the derived quench rate in Figure 24. There is a clear trend of increasing $\gamma_{\mathrm{BH}}$ with increasing $\gamma$. But there is considerable dispersion, and the current black hole growth rate is typically much less than the quench rate. Most of the AGNs in the transition region are accreting far below the Eddington limit (Heckman et al. 2004). This suggests that if the processes of black hole growth and star formation are linked, the timescale for the quenching of black hole growth is considerably shorter than the quenching of star formation.

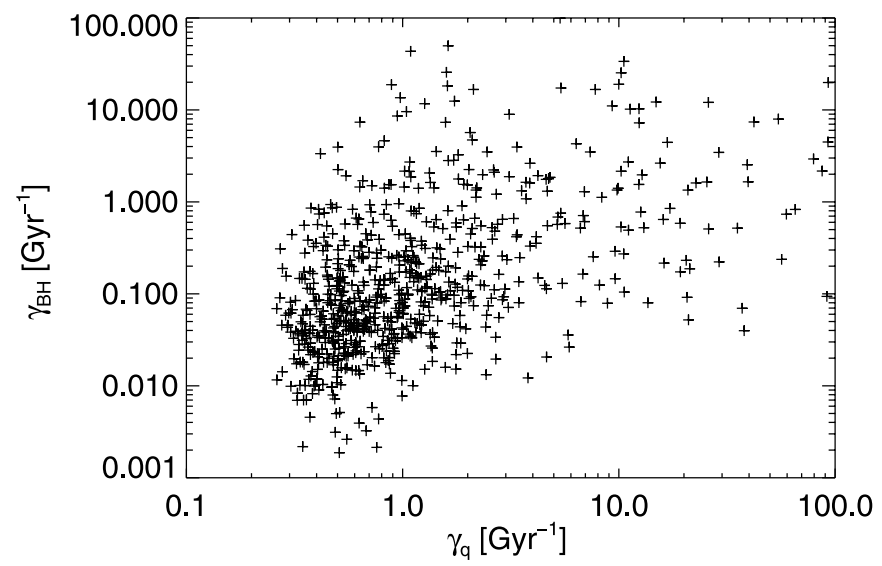

FIG. 24.-Black hole growth rate $\gamma_{\mathrm{BH}}$ (see text) vs. $\gamma_{\text {quench }}$. Color range is $3.75<\mathrm{NUV}-r<4.75$. Absolute magnitude range is $-23<M_{r}<-20$.

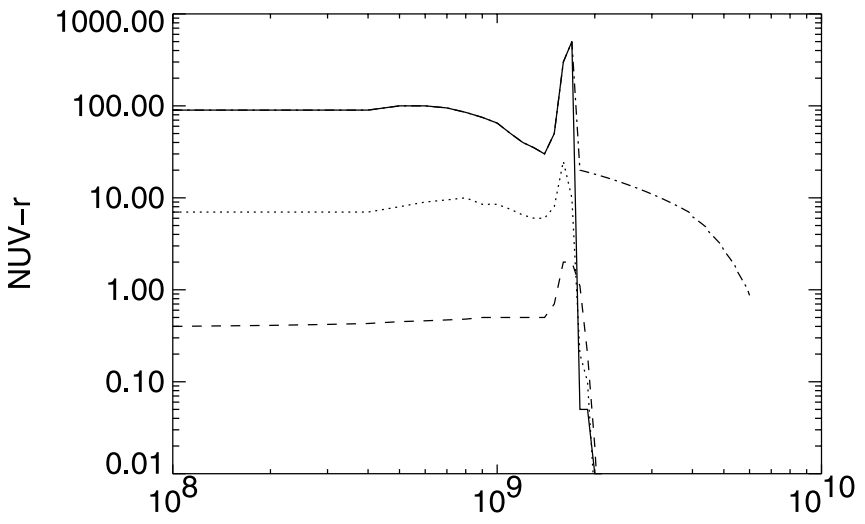

FIG. 25.-Star formation history of simulated major mergers with and without AGN feedback from Di Matteo et al. (2005). Solid line: $v$ (rotation) = $320 \mathrm{~km} \mathrm{~s}^{-1}$, with feedback; dot-dashed line: $v$ (rotation) $=320 \mathrm{~km} \mathrm{~s}^{-1}$, no feedback; dotted line: $v$ (rotation) $=160 \mathrm{~km} \mathrm{~s}^{-1}$, with feedback; dashed line: $v($ rotation $)=80 \mathrm{~km} \mathrm{~s}^{-1}$, with feedback.

Springel et al. (2005) modeled the effects of AGN feedback on the star formation history of a postmerger galaxy. Using their star formation rate histories, we have modeled the color and index evolution in the same fashion as our simple quenched models. In order to make a consistent comparison, we have assumed that their star formation history follows 10 Gyr of constant star formation rate at the starting rate. Figure 25 shows the star formation history. Figure 26 shows the color derivative $d y / d t$ for the four models (3 with AGN feedback and various halo masses and 1 without feedback). Figure 27 shows the loci of models on the $H \delta_{A}-D_{n}(4000)$ plane, compared with the quenched models and the data as in Figure 10. The crosses and diamond (for the no-feedback model) indicate the points when NUV $-r=4.25$. Three of the models have black hole feedback (and three values of dark halo mass), while the fourth shows the most massive object without the feedback effects. Interestingly, there is reasonable overlap between the numerical models and the simple quenched model. These models exhibit a slightly higher value of $H \delta_{A}$. A partial reason is that the numerical models have a large starburst just prior to quenching, which tends to elevate $H \delta_{A}$ [and lower $\left.D_{n}(4000)\right]$ at a given NUV $-r$ color for the fading population. The color derivative for these models at the transitional color is $d y / d t \simeq 3 \mathrm{Gyr}^{-1}$ with feedback and $d y / d t \simeq 0.6 \mathrm{Gyr}^{-1}$ without. A moderate fraction of galaxies show the combination of color,

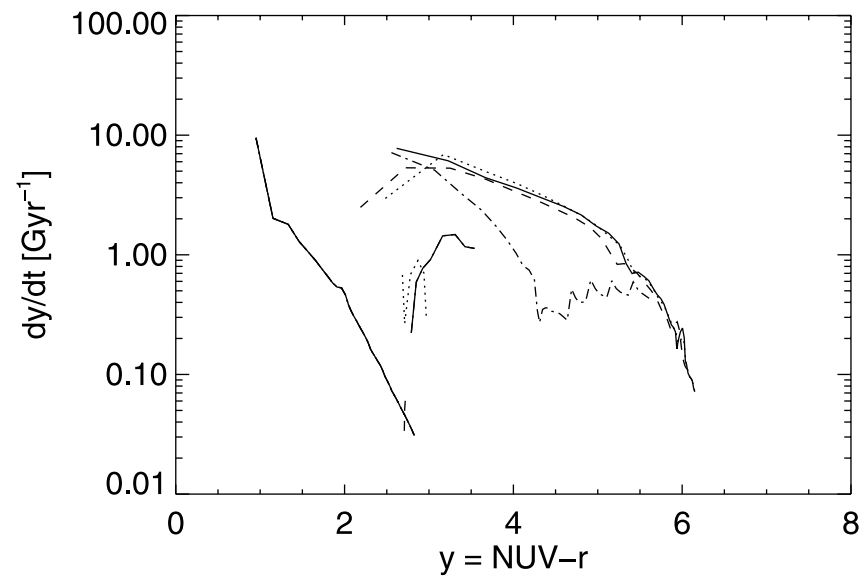

FIG. 26.-Color derivative evolution for the 4 Di Matteo et al. (2005) models. Solid line: $v$ (rotation) $=320 \mathrm{~km} \mathrm{~s}^{-1}$, with feedback; dot-dashed line: $v($ rotation $)=320 \mathrm{~km} \mathrm{~s}^{-1}$, no feedback; dotted line: $v$ (rotation) $=160 \mathrm{~km} \mathrm{~s}^{-1}$, with feedback; dashed line: $v$ (rotation) $=80 \mathrm{~km} \mathrm{~s}^{-1}$, with feedback. 


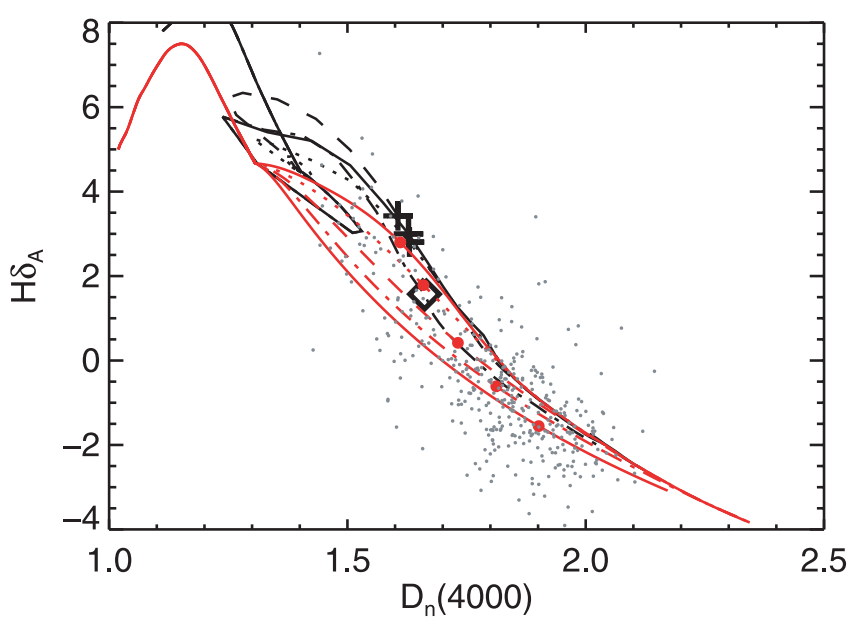

FIG. 27.-Feedback models evolution in $H \delta_{A}$ and $D_{n}(4000)$ plane (black lines). Solid line: $v$ (rotation) $=320 \mathrm{~km} \mathrm{~s}^{-1}$, with feedback; dot-dashed line: $v($ rotation $)=320 \mathrm{~km} \mathrm{~s}^{-1}$, no feedback; dotted line: $v$ (rotation) $=160 \mathrm{~km} \mathrm{~s}^{-1}$, with feedback; dashed line: $v$ (rotation) $=80 \mathrm{~km} \mathrm{~s}^{-1}$, with feedback. Large symbols show values at transition color NUV $-r=4.25$. Pluses show models with feedback, diamond shows model 2 with no feedback. Red curves show simple quenched models for reference.

$H \delta_{A}$ and $D_{n}(4000)$ predicted by the merger/feedback models, while many more show the older indices predicted for mergers and no feedback. But a sizeable fraction show even older indices inconsistent with the numerical histories. Using these tracks to interpret our data leads to lower values of $d y / d t$ for a given combination of index at our transitional color and a correspondingly lower mass flux than we derived above.

Roughly speaking, about $8 \%$ of galaxies (bin 1 of Fig. 13) show properties consistent with the rapid quenching in these major merger models with AGN feedback. Some $10 \%-20 \%$ show properties like the mergers without feedback, and the balance show even slower quenching rates. The majority of galaxies do not exhibit the rapid quench rates predicted by strong AGN feedback. However, it is important to remember that rapid quenches transition rapidly. From Figure 13 (last column), we see that when we correct for the residence time in the transition zone, $50 \%$ of galaxies have quench rates $\gamma \geq 5 \mathrm{Gyr}^{-1}$.
Finally, we can compare these statistics to measurements of the cosmic merger rate. For the redshift range $0.4<z<0.8$, Conselice et al. (2003) obtain a stellar mass accretion rate due to major mergers $\left(M_{B}<-19\right)$ of $1.8 \times 10^{-3} M_{\odot} \mathrm{Mpc}^{-3} \mathrm{Gyr}^{-1}$, which is about $7 \%$ of the total mass flux we measure.

\section{SUMMARY}

We have introduced a new quantity, the mass flux density of galaxies evolving from the blue sequence to the red sequence. We developed a simple technique for constraining this mass flux that exploits the excellent separation of red and blue sequences in the NUV $-r$ band Hess function. We used the volume-corrected number density in the extinction-corrected UV-optical colormagnitude distribution, the stellar age indexes $H \delta_{A}$ and $D_{n}(4000)$, and a simple prescription for spectral evolution using a quenched star formation history. Our estimated mass flux, $\dot{\rho}_{T}=0.033 M_{\odot}$ $\mathrm{yr}^{-1} \mathrm{Mpc}^{-3}$, although strictly an upper limit, compares favorably with estimates of the average mass flux that we make based on the optical and UV data. Galaxies in the transition zone are preferentially AGNs, although we find at best weak evidence that the quench rates are higher in higher luminosity AGNs.

We note that our technique could be applied with alternative evolutionary clocks, such as morphology. Our approach using a single color and spectral index was simple, but a more refined technique would use the entire spectral energy distribution in addition to any relevant spectral indices. Our simple approach is fairly easy to apply to deep galaxy surveys, future work will study the evolution of the mass flux as well as its dependence on galaxy density and environment.

GALEX (Galaxy Evolution Explorer) is a NASA Small Explorer, launched in 2003 April. We gratefully acknowledge NASA's support for construction, operation, and science analysis for the GALEX mission, developed in cooperation with the Centre National d'Etudes Spatiales of France and the Korean Ministry of Science and Technology. We also thank the referee for excellent comments.

\section{Facilities: GALEX}

Balogh, M. L., Baldry, I. K., Nichol, R., Miller, C., Bower, R. G., \& Glazebrook, K. 2004, ApJ, 615, L101

Baldry, I. K., Glazebrook, K., Brinkmann, J., Ivezíc, Z., Lupton, R. H., Nichol, R. C., \& Szalay, A. S. 2004, ApJ, 600, 681

Bell, E. F., McIntosh, D. H., Katz, N., \& Weinberg, M. D. 2003, ApJS, 149, 289

Bell, E. F., et al. 2004, ApJ, 608, 752

Blanton, M. 2006, ApJ, 648, 268

Blanton, M. R., et al. 2003, AJ, 125, 2348

Brinchmann, J., Charlot, S., White, S. D. M., Tremonti, C., Kauffmann, G., Heckman, T., \& Brinkmann, J. 2004, MNRAS, 351, 1151

Bruzual, G., \& Charlot, S. 2003, MNRAS, 344, 1000

Calzetti, D., Kinney, A. L., \& Storchi-Bergmann, T. 1994, ApJ, 429, 582

Conselice, C. J., Bershady, M. A., Dickinson, M., \& Papovich, C. 2003, AJ, 126,1183

\section{EFERENCES}

Di Matteo, T., Springel, V., \& Hernquist, L. 2005, Nature, 433, 604

Faber, S. M., et al. 2007, ApJS, 665, 265

Heckman, T. M., Kauffmann, G., Brinchmann, J., Charlot, S., Tremonti, C., \& White, S. D. M. 2004, ApJ, 613, 109

Johnson, B., et al. 2007a, ApJS, 173, 377 2007b, ApJS, 173, 392

Kauffmann, G. et al, 2003, MNRAS, 341, 33

Pickles, A. J. 1998, PASP, 110, 863

Schiminovich, D., et al. 2005, ApJ, 619, L47

Springel, V., Di Matteo, T., \& Hernquist, L. 2005, ApJ, 620, L79

Tinsley, B. M. 1968, ApJ, 151, 547

Tremaine, S., et al. 2002, ApJ, 574, 740

Wyder, T., et al. 2007, ApJS, 173, 293

Yi, S. K., et al. 2005, ApJ, 619, L111 